\title{
Towards Performance Evaluation of Cognitive Radio Network in Realistic Environment
}

\author{
Vivek Kukreja ${ }^{1}$, Shailender Gupta ${ }^{2}$, Bharat Bhushan ${ }^{3}$ Chander Kumar $^{4}$ \\ YMCA University of Science and Technology \\ dit.vivek@gmail.com ${ }^{1}$, shailender81@gmail.com ${ }^{2}$,bhrts@yahoo.com ${ }^{3}$,nagpalckuma@ $@$ rediffmail.com ${ }^{4}$
}

\begin{abstract}
The scarcity of free spectrum compels us to look for alternatives for ever increasing wireless applications. Cognitive Radios (CR) is one such alternative that can solve this problem. The network nodes having CR capability is termed as Cognitive Radio Network (CRN). To have communication in CRN a routing protocol is required. The primary goal of which is to provide a route from source to destination. Various routing protocols have been proposed and tested in idealistic environment using simulation software such as NS-2 and QualNet. This paper is an effort in the same direction but the efficacy is evaluated in realistic conditions by designing a simulator in MATLAB-7. To make the network scenario realistic obstacles of different shapes, type, sizes and numbers have been introduced. In addition to that the shape of the periphery is also varied to find the impact of it on routing protocols. From the results it is observed that the outcomes in the realistic and idealistic vary significantly. The reason for the same has also been discussed in this paper.
\end{abstract}

Index Terms-Cognitive Radio, Routing Protocols, Mobility, Environment.

\section{INTRODUCTION}

Cognitive Radio Network (CRN) [2-3] is the current wireless technology requirement as it solves the problem of spectrum scarcity. CRN employs two categories of nodes [2-5]: Primary User (PU) and Secondary User (SU). The PU is allocated a license spectrum and can use it whenever it wishes to do so. The SU communicate through the licensed spectrum of PU nodes whenever the channel is not occupied by the PU node i.e. in CR technology the licensed spectrum of existing wireless network is utilized opportunistically. Whenever the PU's become active the SU nodes must give up the communication channel for use by PU and switch to another channel if available. Various researchers have developed routing strategies that have proved beneficial in utilizing spectrum efficiently [16-18]. To evaluate the efficacy of these routing protocols simulation tools such as QualNet, NS2 and Glomosim are used. The simulation tool [6-7] provides a platform with specific advantage over real world as follows:

\section{A. Repeatable scenarios}

The protocol developers require changes to the protocol and retest the protocol in the same scenario. Hence this feature aids in deeper understanding of how the changes impact the performance of protocol. To implement this feature in real world, significant work needs to be performed.

\section{B. Isolation of parameters}

The feature helps the protocol designers to find the impact of single parameter such as mobility; bandwidth and transmission range [5-8], while keeping the other parameters constant. This feature is quite difficult to implement in real world.

\section{Variety of scenarios and network configurations}

The feature helps the protocol designers to change the scenario properties such as: number of nodes can be altered or data link layer protocol can be changed. Implementing this feature in real world requires much more duration and labor.

All of these advantages are not available with real world experiments. Due to these benefits, simulation tool has become a popular tool for the development. An important component of the simulators is the simulation region in which the nodes are to be deployed. The simulation region may contain obstacles [8-9] of varying shape and may contain varying number of obstacle. Moreover the obstacle may be of different types for instance river and mountain type. The former type obstacle restricts node movement only where-as the latter one restricts node movement as well as effective transmission range of the nodes. In addition to above, another factor of simulator is the shape of the simulation region in which the nodes are to be deployed. All these factors significantly affect the performance of routing protocols. Therefore to make the study exhaustive following experiments are performed.

\section{Experiment 1}

Evaluating the Impact on network performance by considering obstacles of different shapes in the simulation region. 


\section{E. Experiment 2}

Evaluating the Impact on network performance considering obstacles of different types in the simulation region.

\section{F. Experiment 3}

Evaluating the Impact on network performance by considering obstacles of different sizes within the simulation region.

\section{G. Experiment 4}

Evaluating the Impact on network performance by considering the obstacles and varying the shape of simulation region.

To perform these experiments a simulator was designed in MATLAB-7. The paper also discusses the reasons for the variations in results in realistic and idealistic environment. The rest of the paper is organized as follows: Section 2 Illustrates the Literature Survey. Section 3 gives Algorithm and simulation set up parameters. Section 4 provides the impact of realistic scenario on CRN. Section 5 present the conclusion followed by the references.

\section{LITERATURE SURVEY}

There are only few researchers who have tried to make simulation as realistic as possible. Their work is as follows.

The work done by Amit Jardosh et. al. [8] was the first in the direction of making the scenario realistic. They incorporated obstacle in the simulation region of ad hoc network and observed that the use of obstacle or realistic condition not only reduces packet delivery ratio but at the same time increases the end to end delay of data packets.

A different strategy was adopted by $\mathrm{N}$ Meghanathan [9] to make the scenario realistic. Their paper showed that the performance of the ad hoc network starts deteriorating as the skewed ness of periphery from square to rectangle increases i.e. as the length to breadth ratio increases the performance of ad hoc network falls significantly.

Further comprehensive study on the shape of the simulation area and type of the obstacle was carried out by C. K. Nagpal et. al [1]. According to their observation, not only the shape of the periphery but the type (mountain or river) and number of obstacle also influences the performance of routing protocols of ad hoc network.

All these mechanism have been developed for ad hoc network and none of the researcher has made an attempt to incorporate realistic conditions in CRN environment. Thus this paper is written by keeping the following objectives in mind.

1. To make the scenario realistic by incorporating obstacles of different types and analyze the cause of the same on performance of CRN.
2. To vary the shape of the periphery and analyze the cause of the same on performance of CRN.

3. To show that realistic results are quite different from idealistic ones

4. To vary the concentration as well as time allocated by PU's to SU's in realistic condition thus showing the need for CRN.

We are of the opinion that before deploying any protocol, the geographical shape and the obstacle shape as well as number of obstacle in the region should be incorporated in the simulation software so that a true picture can be obtained about the protocol which is best suited under the given environmental conditions. The next section discusses the experiments performed by us to study the effect of same on the efficacy of routing protocols.

\section{SIMULATION AND ALGORITHM}

\section{A. Performance Metrics}

The following metrics has been used to evaluate the performance of CRN in the idealistic and realistic ones.

- Packet Delivery Ratio (PDR)-Defined as the ratio of total packets received by different destinations to the total number of packets transmitted by various source nodes.

- Hop Count- Defined as the number of intermediate hops from source to the destination.

- Path Optimality- Defined as the ratio of total distance traversed in cognitive network to the distance traversed in a network having no PU nodes.

In the next section an algorithm is used to calculate these performance metrics.

\section{B. Algorithm}

The algorithm 1 helps in calculating the above mentioned metrics. In the algorithm initially total 40 nodes are considered as SU, and the PU nodes are varied as fraction of SU from $0 \%$ to $50 \%$ in steps of $10 \%$ for each run.

A variable count is used to count the number of paths formed. If path exists between S-D pair the value of count variable is incremented by 1 . For calculating the value of average Hop Count the Cum_Hop_count variable is used (initialized to zero). Initially check for shortest path from source to destination path, If path exists check whether the path is devoid of obstacles, if path determine is intercepted by any obstacle, search for alternate paths devoid of obstacles, if no such path without obstacles is feasible search for next destination. This process is repeated for all S-D pairs. For calculating PDR the source sends 100 packets using procedure send_data() between every S-D pair and returns successfully packets received by destination. A variable called Cum_Data_packet is used to find cumulative value of packet received by destination. Path_length contain the distance between source and destination. The 
average Hop Count, PoR and packet delivery ratio are calculated by using formula given in Algorithm.

Algorithm 1: To calculate performance metrics

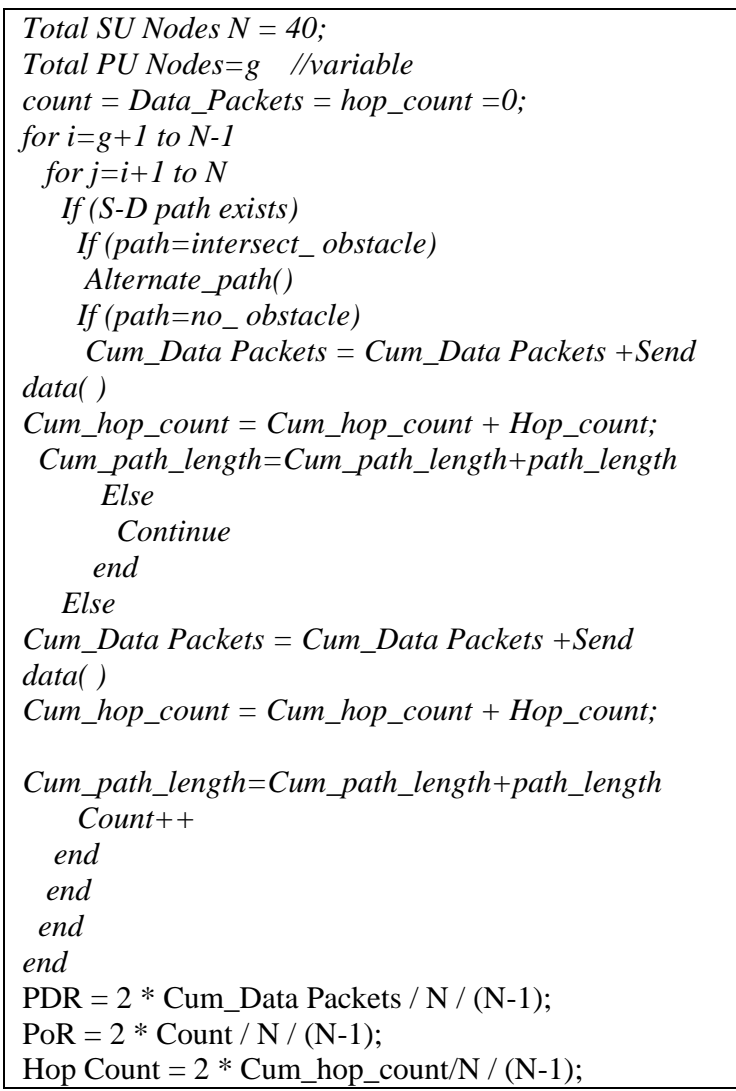

\section{Set up Parameters}

Table 1 shows the values of set up parameters used for simulation purpose.

\begin{tabular}{|c|c|}
\hline \multicolumn{2}{|c|}{ TABLE1: SET Up PARAMETERS } \\
\hline Set up parameter & Value \\
\hline $\begin{array}{l}\text { Area of simulation } \\
\text { Region }\end{array}$ & 2000x2000 sq units \\
\hline Number of SU nodes & 40 \\
\hline Numbers of PU nodes & $\begin{array}{c}\text { Varied from } 5 \% \text { to } 50 \% \text { of SU } \\
\text { nodes }\end{array}$ \\
\hline Transmission Range & $300 \mathrm{~m}$ \\
\hline Mobility Model & Random Walk \\
\hline PU nodes mobility & NIL, PU nodes are fixed \\
\hline Speed of SU nodes & $15 \mathrm{~m} / \mathrm{s}$ \\
\hline Obstacle shape & $\begin{array}{c}\text { Rectangle } \\
\text { Square } \\
\text { Ellipse } \\
\text { Circle } \\
\end{array}$ \\
\hline Nodes position & Random \\
\hline Routing algorithm & Dijkstra's Shortest Path \\
\hline $\begin{array}{l}\text { Packet transmission } \\
\text { interval }\end{array}$ & $1 \mathrm{sec}$ \\
\hline Packet Size & 512 bytes \\
\hline Number of packet sent & 100 \\
\hline Obstacle type & $\begin{array}{c}\text { Mountain } \\
\text { River }\end{array}$ \\
\hline Area of obstacle & $\begin{array}{l}200,000 \text { sq units } \\
400,000 \text { sq units } \\
600,000 \text { sq units } \\
800,000 \text { sq units }\end{array}$ \\
\hline Shape of Region & $\begin{array}{c}\text { Square } \\
\text { Circle } \\
\text { Ellipse } \\
\text { Rectangle }\end{array}$ \\
\hline
\end{tabular}

\section{Experiment 1}

In this experiment we incorporated different shapes (Square, Rectangle, Square and Ellipse) of the obstacle in the simulation region to make the simulation realistic for CRN. In addition to it, number of PU's and time allotted by PU to SU's was also varied so as to show the need for CRN. Various snapshots of the simulation region are shown from Figure1 (a) to Figure1(d).

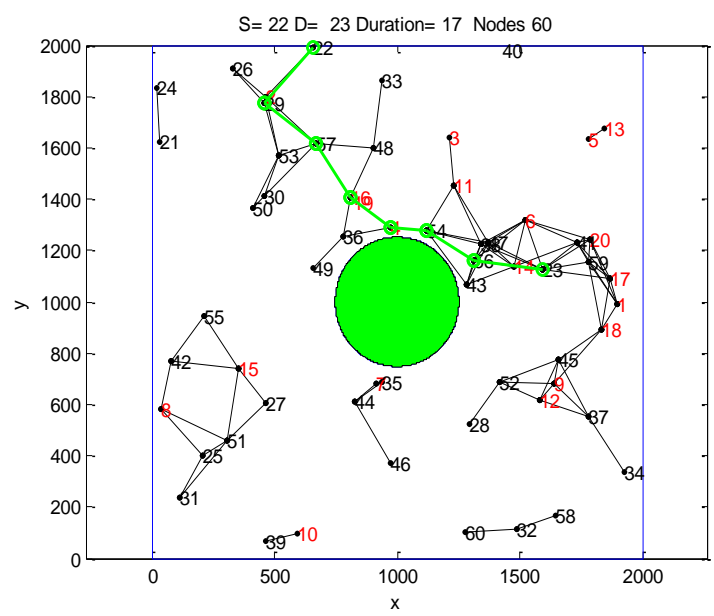

Figure 1(a). Circular obstacle 


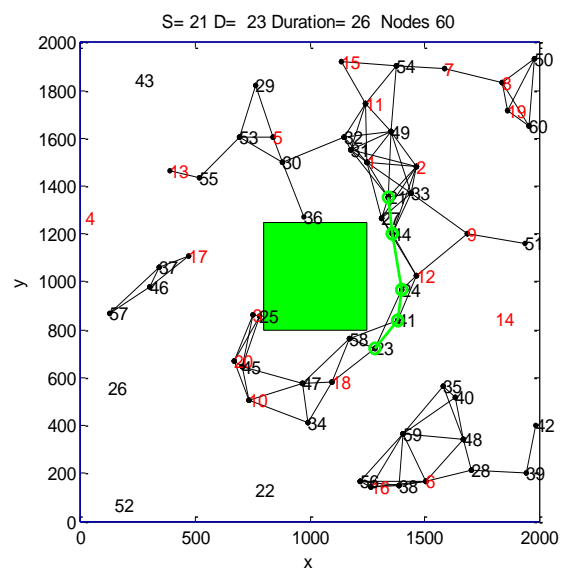

Figure 1(b). Square obstacle

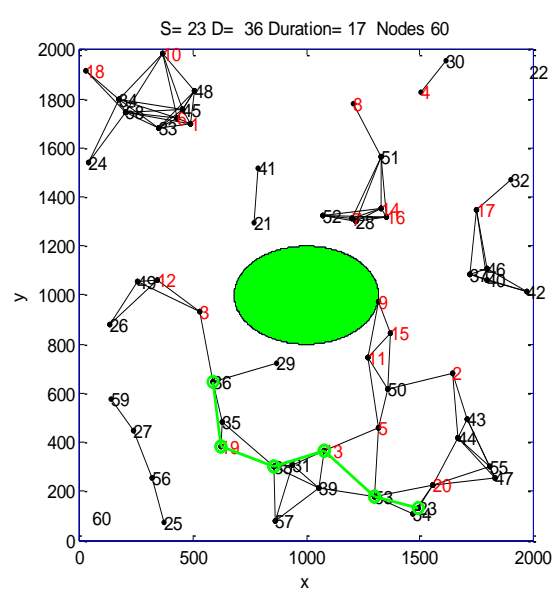

Figure 1(c). Ellipse obstacle

\section{D.1. PDR Comparison}

Figure 1.1 to Figure 1.4 shows the graphs for different shapes of the obstacle. From the results following inference can be drawn:

- $\quad$ By increasing the PU Concentration and Increasing the PU activation time the value of PDR increases significantly.

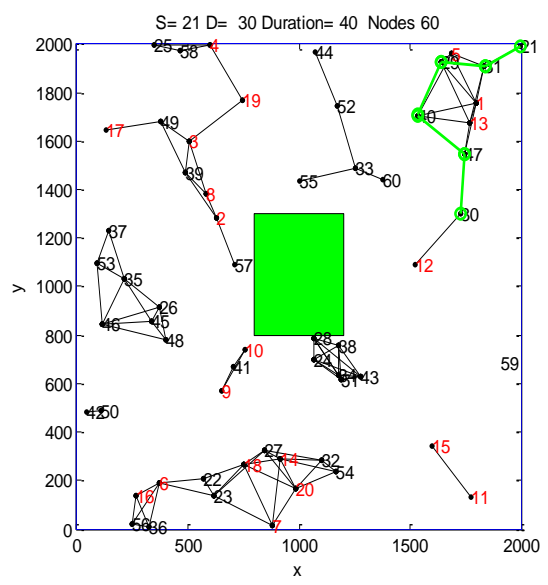

Figure 1(d). Rectangle obstacle
- The value of the PDR with obstacles is found to be highest for circular shaped obstacle.

- The slope of the graphs in case of circular region is highest.

- PDR curve for the Ellipse and square regions are approximately same. the PDR curve for ellipse approaches the PDR curve for the square region on the cost of increased Hop Count

- PDR in case of rectangular obstacle is least, which is due to increase in the Hop Count and Path length.

- Slope of curves is least in the case of rectangular obstacle.

- Fluctuations in the PDR curve for Rectangular obstacle are maximum i.e. longer the length of path more is the probability of break in the path.

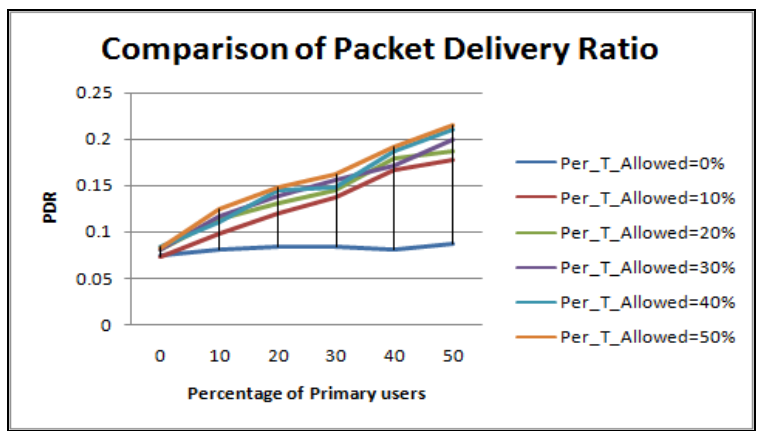

Figure 1.1. Impact on PDR in Circular obstacle situation

\section{2 Hop Count Comparison}

Figure 1.5 to Figure 1.8 shows the hop count value for different shapes. These curves justifies the need of CRN.

The following inference can be drawn from the graphs:

- Hop count value is maximum in case of rectangular shaped obstacle.

- In comparison to other shape of the obstacle the fluctuations in value for rectangular obstacle is maximum.

- $\quad$ The slope for Hop Count in case of ellipse and square shapes is approximately same.

- As the concentration of PU nodes is increased from 0 to $10 \%$, the variation in the value of hop count is negligible.

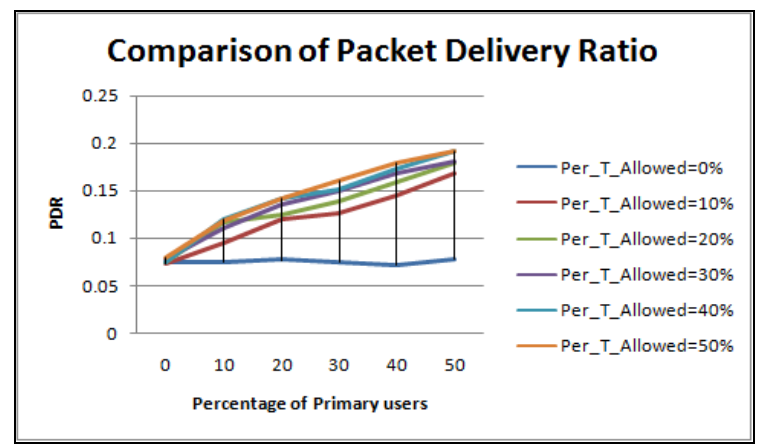

Figure 1.2. Impact on PDR in Square obstacle situation 


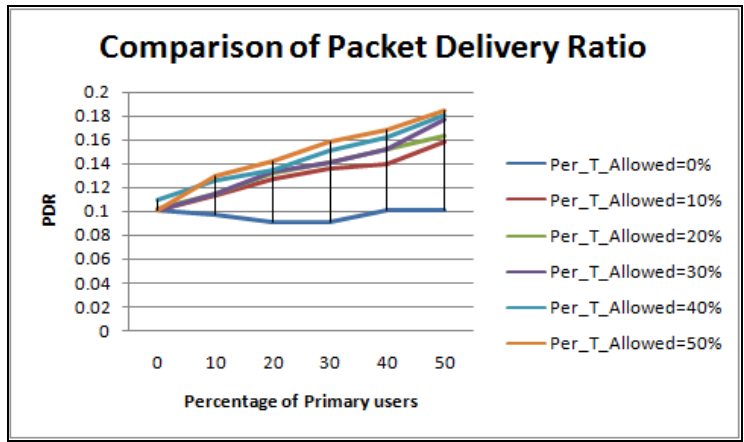

Figure 1.3. Impact on PDR in Elliptical obstacle situation

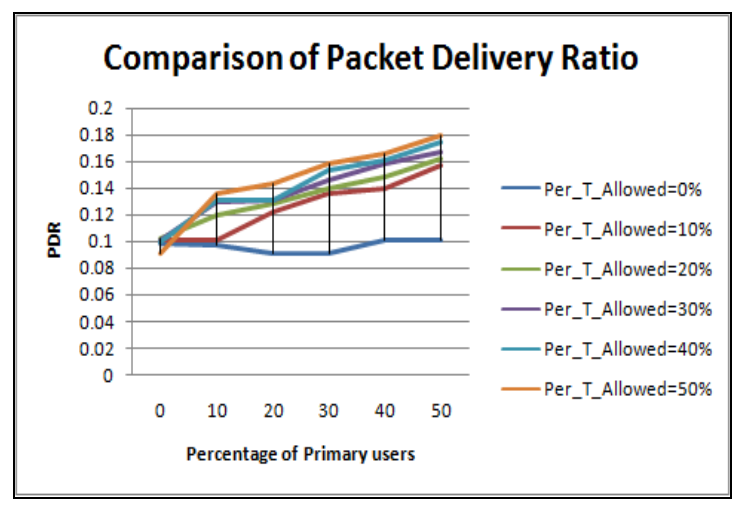

Figure 1.4. Impact on PDR in Rectangle obstacle situation

\section{D.3. Path optimality comparison}

Figure 1.9 to Figure 1.12 shows the path optimality values for different shape of the obstacle.

The following inference can be obtained about path optimality values:

- For all the shapes of the obstacles it can be seen that path length increases with increase in number of PU users.

- $\quad$ Maximum value of Path Optimality is obtained in the case of Rectangular obstacle. Thus it shows that in case of rectangular obstacle the path will not be reliable and hence its PDR value will be lesser in comparison to other shape of the obstacle.

- The fluctuations in case of rectangular and elliptical shape obstacles are greater as more is the path length more will be the likelihood of path break.

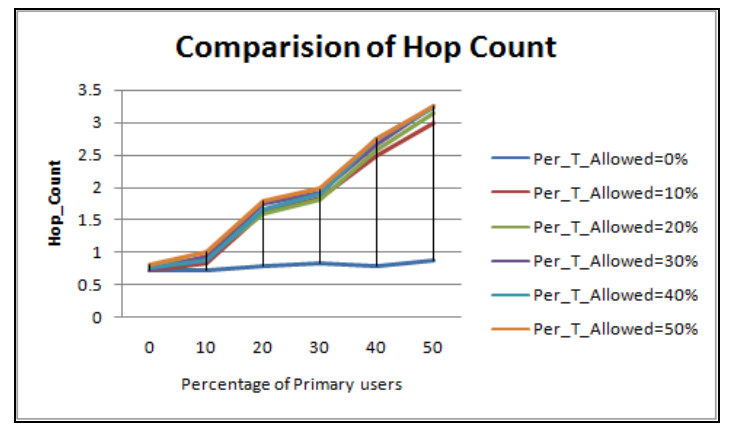

Figure 1.5. Impact on Hop Count in Circular obstacle situation

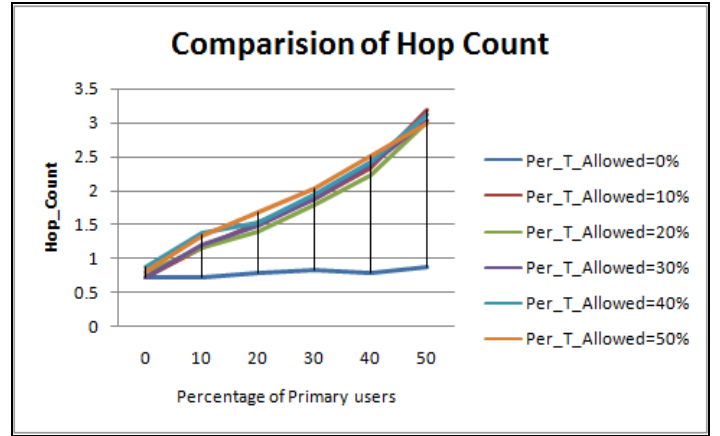

Figure 1.6. Impact on Hop Count in square obstacle situation

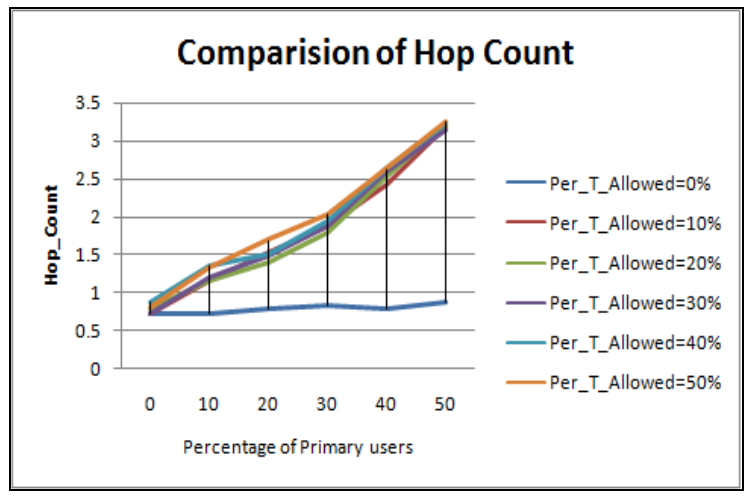

Figure 1.7. Impact on Hop Count ellipse obstacle situation

\section{E. Analysis of Experiment 1}

It can be seen from the Table 2 that best results are obtained in case of circular obstacle followed by square, ellipse and then rectangle. The reason for the same is the symmetry of the obstacle. As it is well known fact that "symmetry leads to stability" .Symmetry can be measured in terms of lines of symmetry i.e. a line that divides the figure in to two congruent parts.

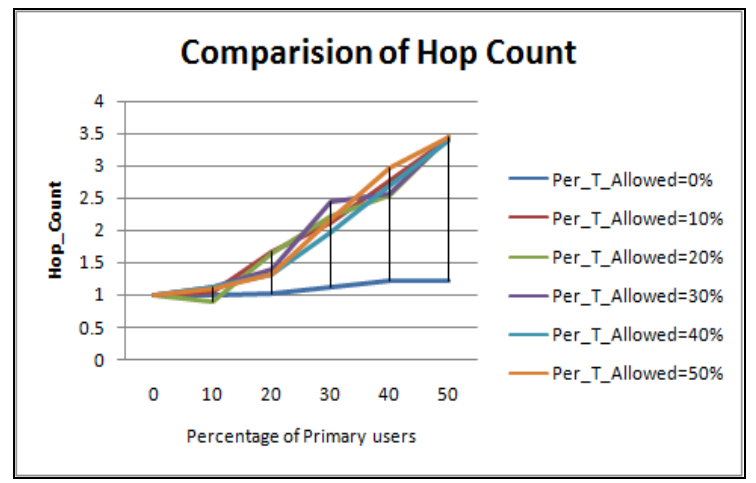

Figure 1.8. Impact on Hop Count in rectangle obstacle situation 


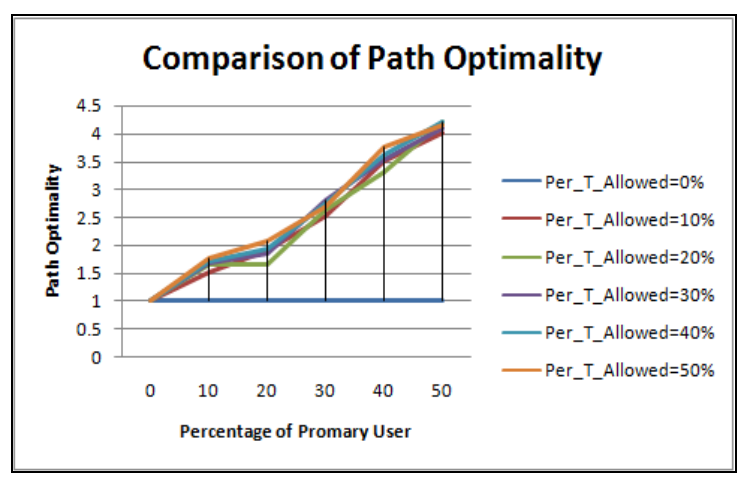

Figure 1.9. Impact on Path Optimality in Circular obstacle situation

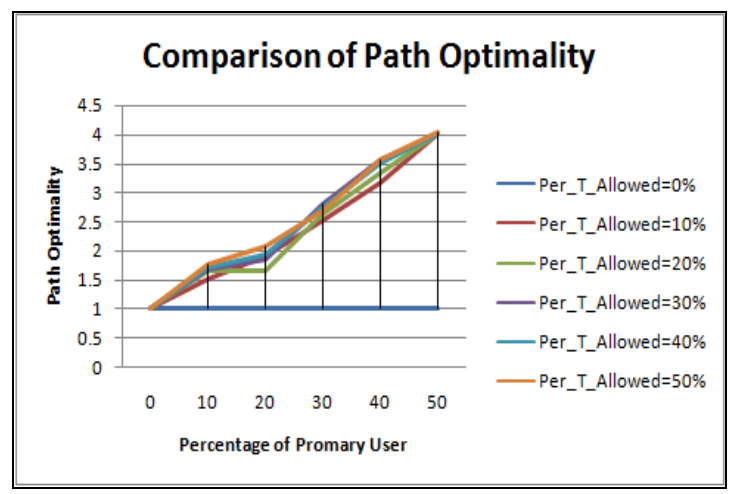

Figure 1.10. Impact on Path Optimality in square obstacle situation

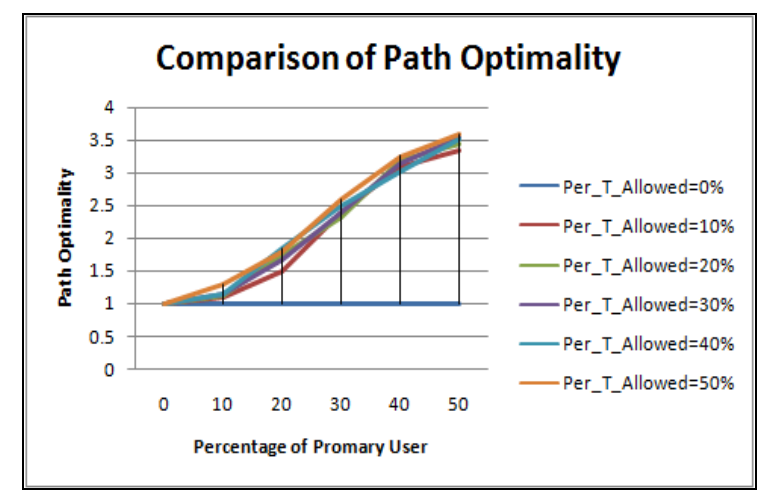

Figure 1.11. Impact on Path Optimality in elliptical obstacle situation

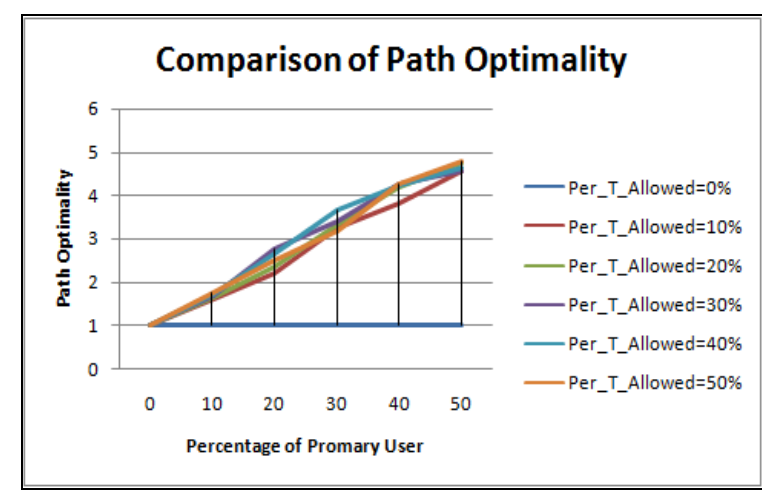

Figure 1.12. Impact on Path Optimality in Rectangular obstacle situation
The obstacle shapes having more number of lines of symmetry will offer less hindrance. As observed in Table 2, Circular shape has highest lines of symmetry, while Ellipse and rectangle shapes have least number of lines of symmetry, consequently the hindrance in Elliptical and rectangular shapes will be maximum. This implies that the results of elliptical and rectangular obstacle should be same but the periphery of the rectangular shape obstacle is greater in comparison to elliptical shape. This leads to greater obstruction offered by the rectangular shape. Hence we can say that the results of hop count are in the increasing order of circle, square, ellipse and rectangular obstacle.

\section{F. Experiment 2}

In this experiment we consider different type of obstacle such as river and Mountain. The former type obstacle i.e., river type will restrict the movement of nodes but the packets can be exchanged between the nodes present on either side of the river as also evident in the snapshot. On the other hand mountain type obstacle not only restricts the node movement but also cuts the communication otherwise possible between the nodes lying that are within the transmission range of each other.

\section{F.1.PDR Comparison}

Following Inference can be drawn from the Figure 2.1 and Figure 2.2

- In case of river type obstacle the PDR value is higher in comparison to its value in mountain type obstacle.

- Increased fluctuation in PDR value for mountain type obstacle due to longer path lengths.

TABLE 2: OVERALl COMPARISON OF EXPERIMENT 1

\begin{tabular}{|c|c|c|c|c|}
\hline $\begin{array}{l}\text { Performance } \\
\text { Metrics } \\
V_{\text {Shape of }}^{\rightarrow} \\
\text { Obstacle }\end{array}$ & Circle & Square & Ellipse & Rectangle \\
\hline $\begin{array}{c}\text { Lines of } \\
\text { Symmetry }\end{array}$ & $\begin{array}{c}\text { Infinit } \\
\mathrm{e}\end{array}$ & 4 & 2 & 2 \\
\hline $\begin{array}{l}\text { PDR } \\
\text { Min } \\
\text { Max }\end{array}$ & $\begin{array}{l}0.08 \\
0.21\end{array}$ & $\begin{array}{l}0.07 \\
0.19\end{array}$ & $\begin{array}{l}0.08 \\
0.18\end{array}$ & $\begin{array}{l}0.09 \\
0.18\end{array}$ \\
\hline $\begin{array}{c}\text { Path } \\
\text { Optimality } \\
\text { Min } \\
\text { Max }\end{array}$ & $\begin{array}{c}1 \\
4.15\end{array}$ & $\begin{array}{c}1 \\
4.05\end{array}$ & $\begin{array}{c}1 \\
3.4\end{array}$ & $\begin{array}{c}1 \\
4.5\end{array}$ \\
\hline $\begin{array}{l}\text { Hop count } \\
\text { Min } \\
\text { Max }\end{array}$ & $\begin{array}{l}0.78 \\
3.25\end{array}$ & $\begin{array}{l}0.71 \\
3.11\end{array}$ & $\begin{array}{l}0.74 \\
3.14\end{array}$ & $\begin{array}{l}0.73 \\
3.44\end{array}$ \\
\hline
\end{tabular}




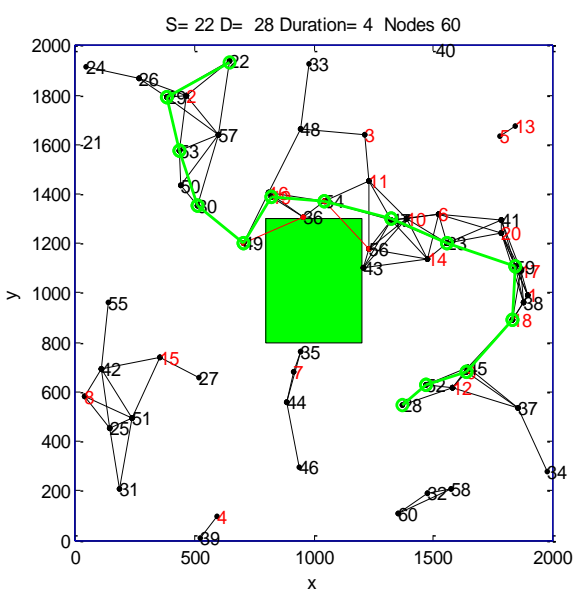

Figure 2(a). Mountain type obstacle

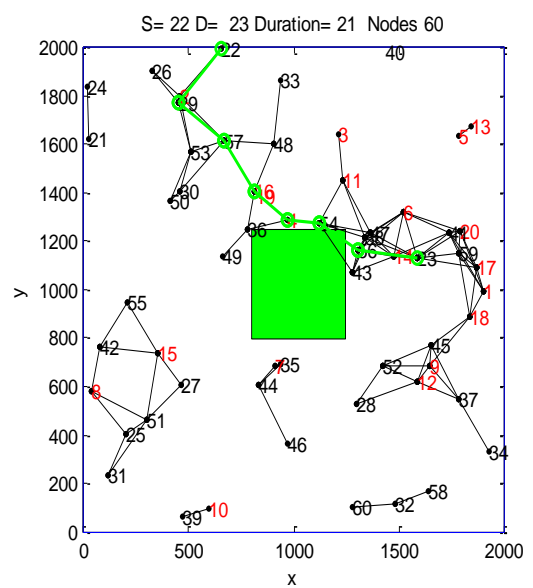

Figure 2(b). River type obstacle

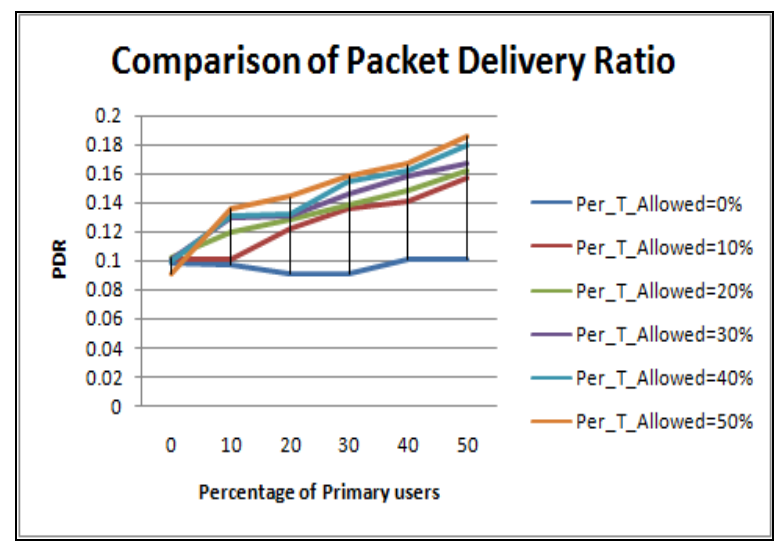

Figure 2.1. Impact on PDR in Mountain type obstacle situation

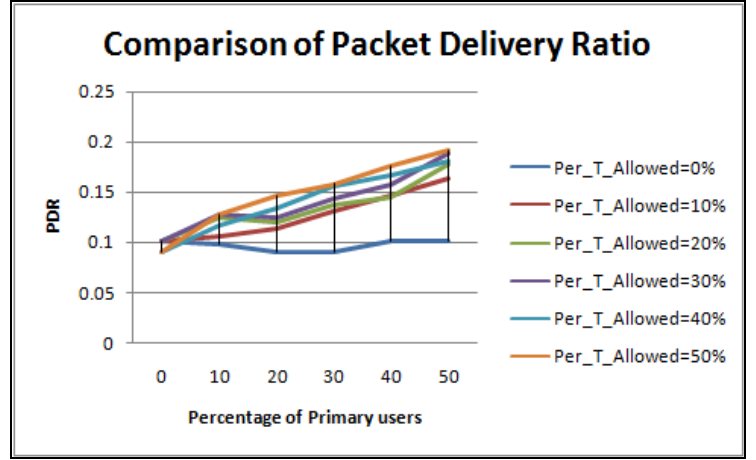

Figure 2.2. Impact on PDR in River type obstacle situation

\section{F.2. Hop Count Comparison}

Figure 2.3 and Figure 2.4 show the hop count comparison for different types of the obstacles. The following inference can be drawn:

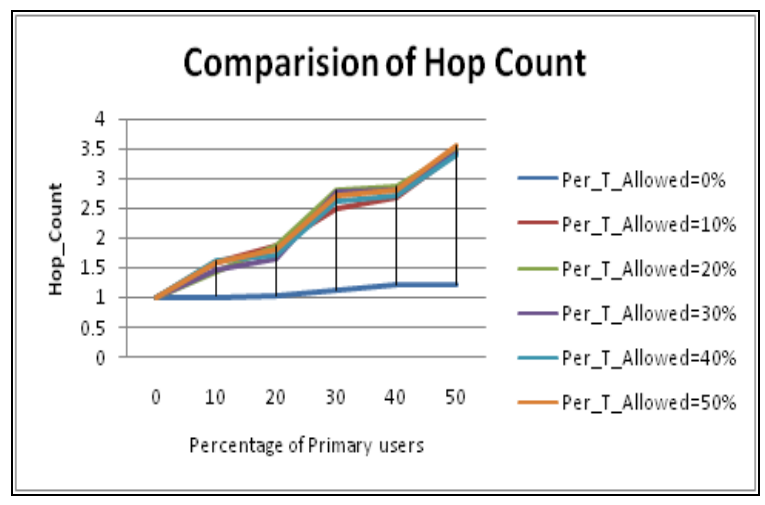

Figure 2.3. Impact on Hop Count in Mountain type obstacle situation

- The value of Hop Count is large for mountain type obstacle in comparison to river type obstacle as observed in Figure 2.3 and Figure 2.4.

- In case of mountain type obstacle the hop count value is consistently increasing for all values of PU, but in case of river type obstacle it is not so for the beginning concentration of $\mathrm{PU}(0$ to $10 \%)$.

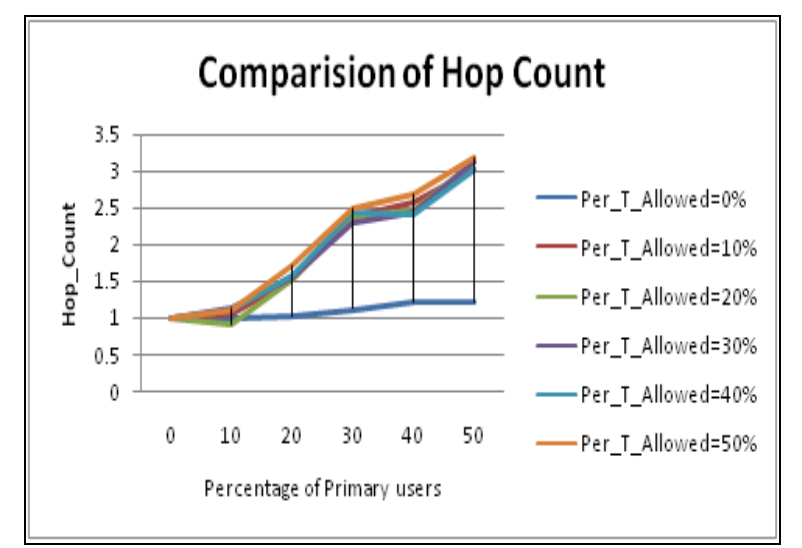

Figure 2.4. Impact on Hop Count in River type obstacle situation 


\section{F.3. Path Optimality Comparison}

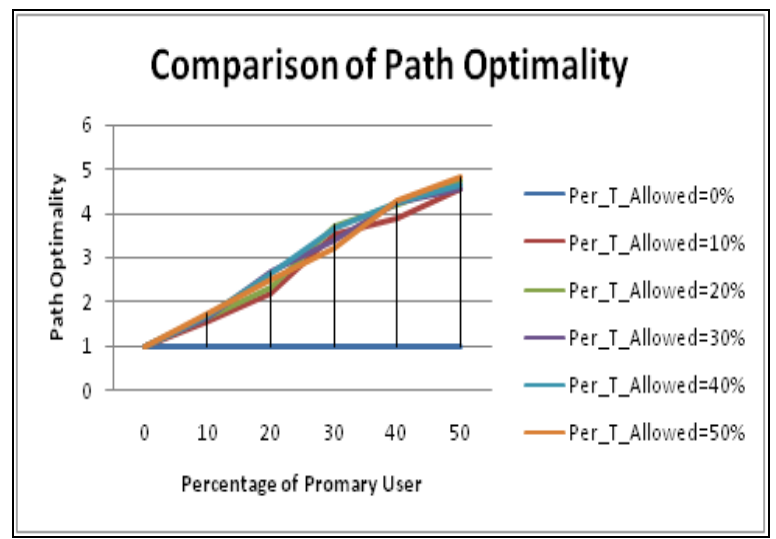

Figure 2.5. Impact on Path Optimality in Mountain type obstacle situation

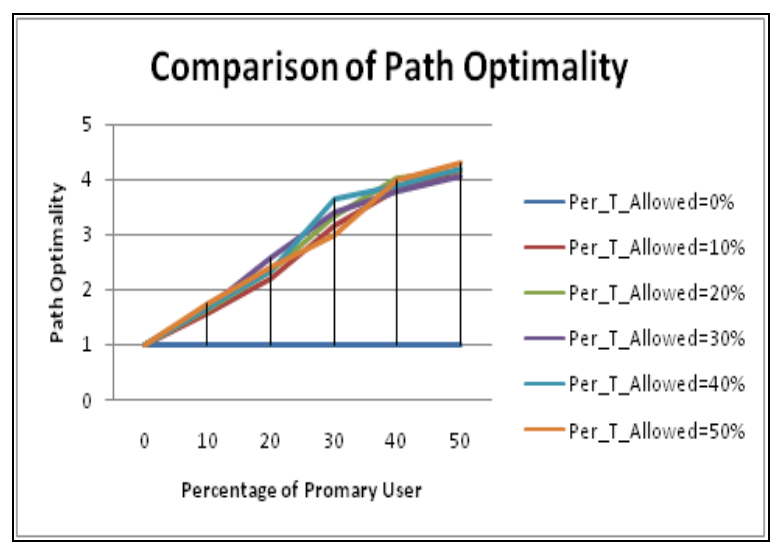

Figure 2.6. Impact on Path Optimality in River type obstacle situation

- In the mountain type obstacle deployment, the maximum value of Path Optimality is greater as the paths formed will be longer due to presence of obstacles.

- $\quad$ Fluctuations in the River type are more as paths formed are both around the obstacle as well as through the obstacle.

- $\quad$ Fluctuations in the mountain type are less as paths formed will be around the obstacle only.

\section{G. Analysis of experiment 2}

Before discussing its analysis we would like to give some brief facts as follows:

- $\quad$ More is the path length higher are the chances of path breakage

- A reliable path will certainly have more value of PDR as its path length or hop count shall be small

The experimental result shows that in case of mountain the value of hop count is greater in comparison to river type obstacle since mountain type obstacle not only restricts node movement but also reduces effective transmission range of nodes i.e. it restricts the direct line of sight communication (see Figure 2(a)). As discussed the river type obstacle has lower value of path length hence path will be quite reliable in comparison to mountain type obstacle. Therefore the value of PDR is high in river type obstacle.

TABLE 3: OVERALL COMPARISON OF EXPERIMENT2

\begin{tabular}{c|c|c}
\hline $\begin{array}{c}\text { Performance } \\
\text { Metrics } \\
\text { Type of } \\
\text { Obstacle }\end{array}$ & Mountain & River \\
\hline PDR & 0.09 & 0.09 \\
Min & 0.18 & 0.19 \\
Max & 1 & 1 \\
\hline Path Optimality & 4.8 & 4.2 \\
Min & 1.18 & 0.94 \\
Max & 3.53 & 3.18 \\
\hline Hop count &
\end{tabular}

\section{H. Experiment 3}

In this experiment an effort has been made to find impact of varying obstacle size on performance of CRN is observed. For this purpose a square obstacle is taken and its size is varied from $5 \%$ to $20 \%$ of the size of simulation region with step size of $5 \%$. Figure 3(a) to Figure 3(d) gives the snapshot for varying size of obstacle.

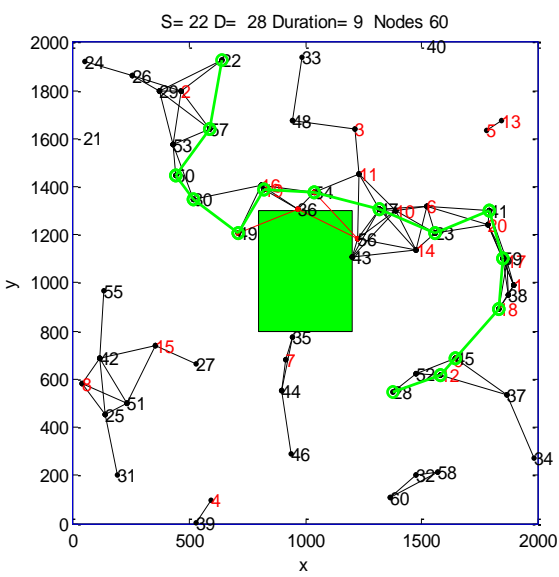

Figure 3(a). Size of obstacle 5\%

\section{H.1. PDR Comparison}

For this experiment we have considered mountain type obstacle. Figure 3.1 to Figure 3.4 displays the Variation in the value of PDR for different size of obstacle, with increase in concentration of Primary users. The following inferences can be drawn from the results.

- As the size of the obstacle increases the effective transmission range of nodes decreases therefore the value of PDR decreases. It is also observed that the maximum value of PDR among all the cases is highest for $5 \%$ size of obstacle.

- When the conditions of PU concentration and PU activation time are subjected; response is maximum for $5 \%$ obstacle size case. 


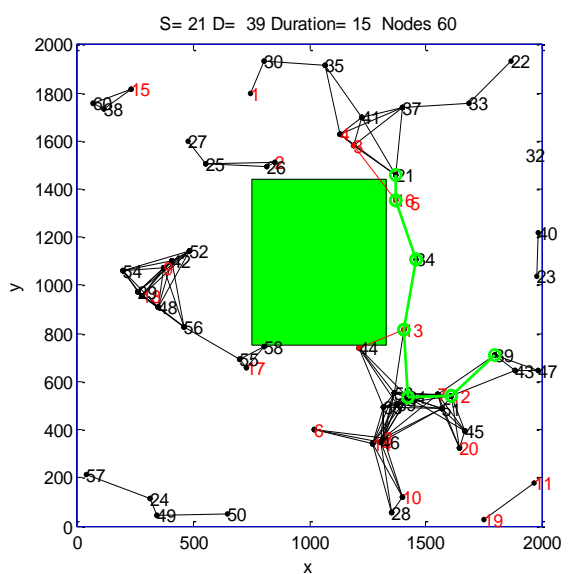

Figure 3(b). Size of obstacle 10\%

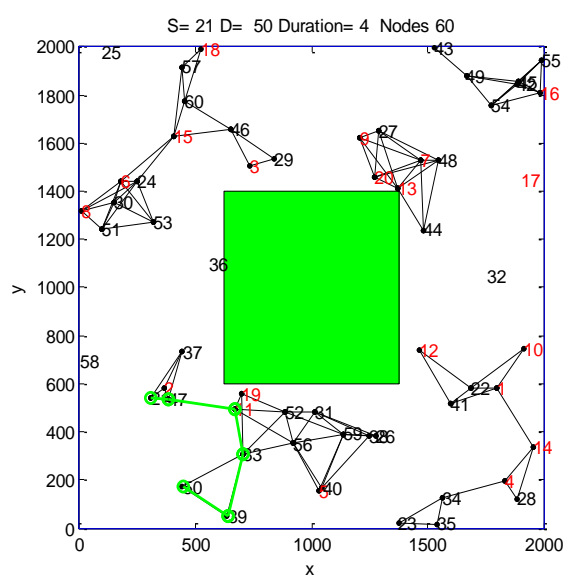

Figure 3(c). Size of obstacle $15 \%$

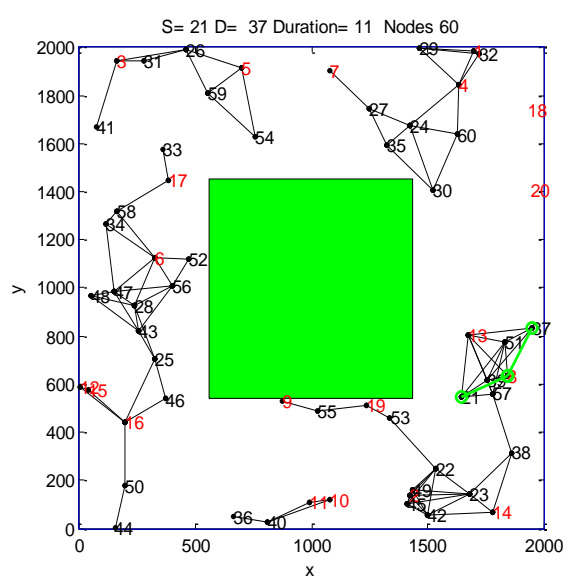

Figure 3(d). Size of obstacle $20 \%$

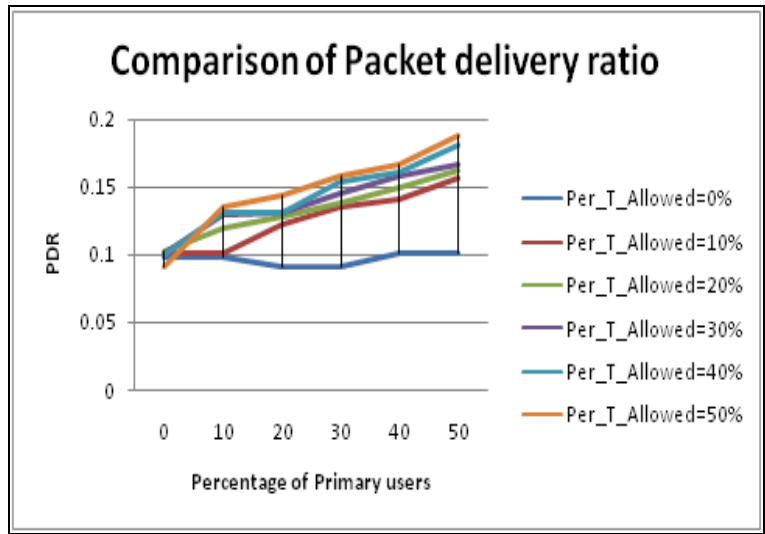

Figure 3.1. Impact on PDR for 5\% size obstacle situation

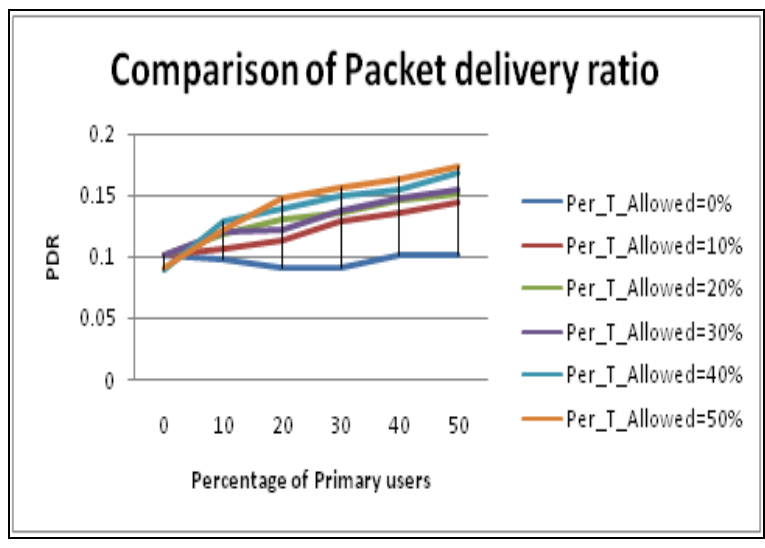

Figure 3.2. Impact on PDR for $10 \%$ size obstacle situation

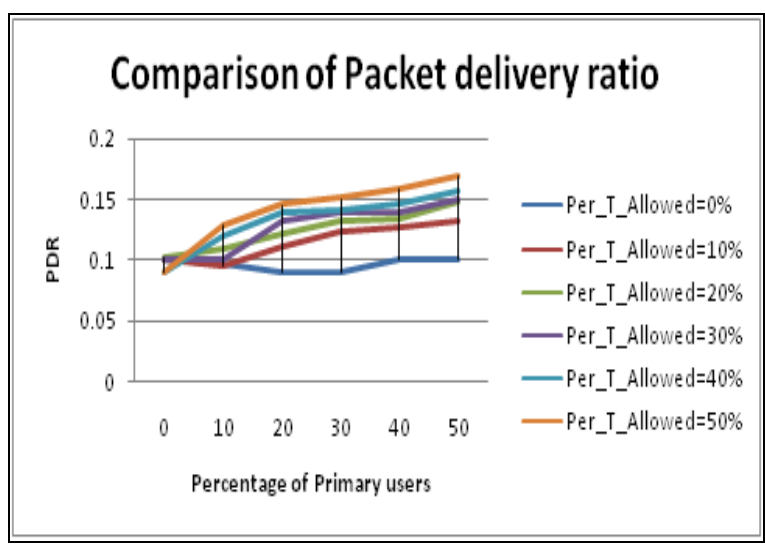

Figure 3.3. Impact on PDR for $15 \%$ size obstacle situation

\section{H.2. Hop Count Comparison}

Figure 3.5 to Figure 3.8 show the impact on Hop Count for varying size of obstacle with increase in PU concentration. Following inference can be drawn:

- It can be easily seen that maximum hop count as well as path optimality value among all cases is largest for 5\% size. On analysis it was found that the successful path formed in case of $20 \%, 15 \%$ and $10 \%$ size of obstacles were quite low since the obstacle free 
region that can be used for communication reduces as a result of which the value of hop count further reduces.

- Fluctuations in the values in the graphs are due to the randomness in the deployment of nodes in the simulation region.

- Little variations is observed for 0 to $20 \%$ of nodes in values of Hop Counts in the graph for $20 \%$ size of obstacle upto $20 \%$.

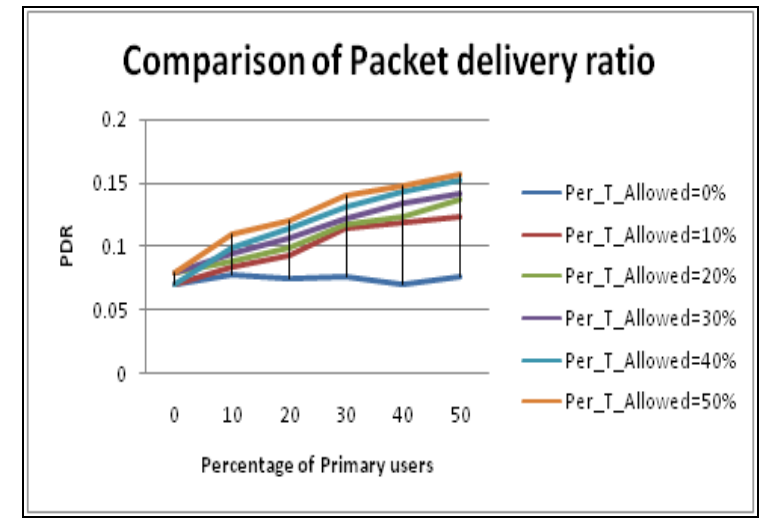

Figure 3.4. Impact on PDR for $20 \%$ size obstacle situation

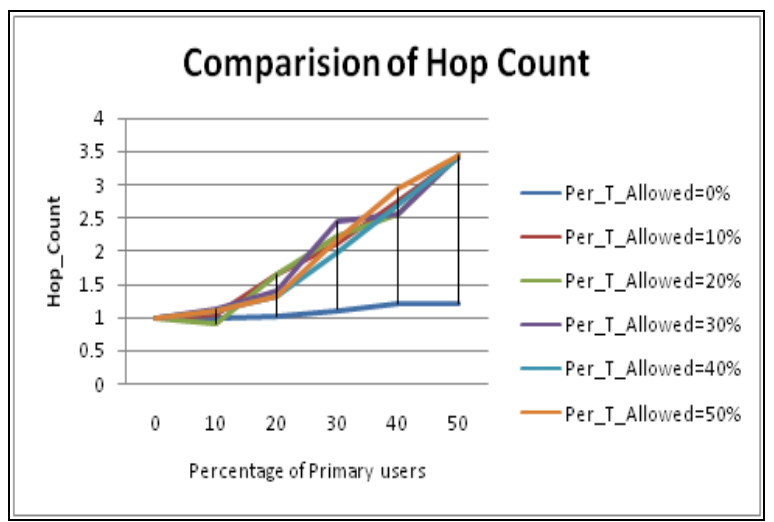

Figure 3.5. Impact on Hop Count for 5\% size obstacle situation

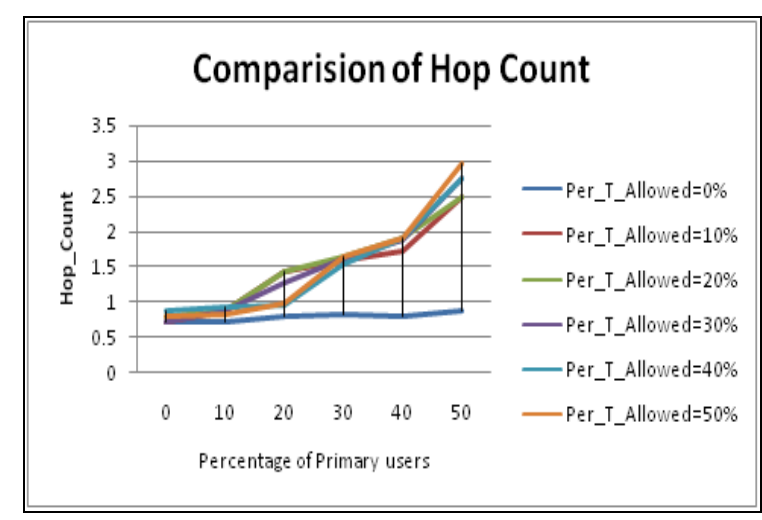

Figure 3.6. Impact on Hop Count for $10 \%$ size obstacle situation

\section{H.3. Path Optimality Comparison}

Figure 3.9 to Figure 3.12 shows the impact on path optimality for varying size of obstacle with rise in PU concentration. Following inference can be drawn:

- The maximum value of Path Optimality is obtained in case of $5 \%$ obstacle size. The reason for the same has been discussed above.

- $\quad$ For $15 \%$ and $20 \%$ size of obstacles, hop count change is negligible when PU concentration is raised from $10 \%$ to $20 \%$.

- $\quad$ For $20 \%$ size obstacle change in the Hop Count value is small when PU concentration is raised from $30 \%$ to $40 \%$.

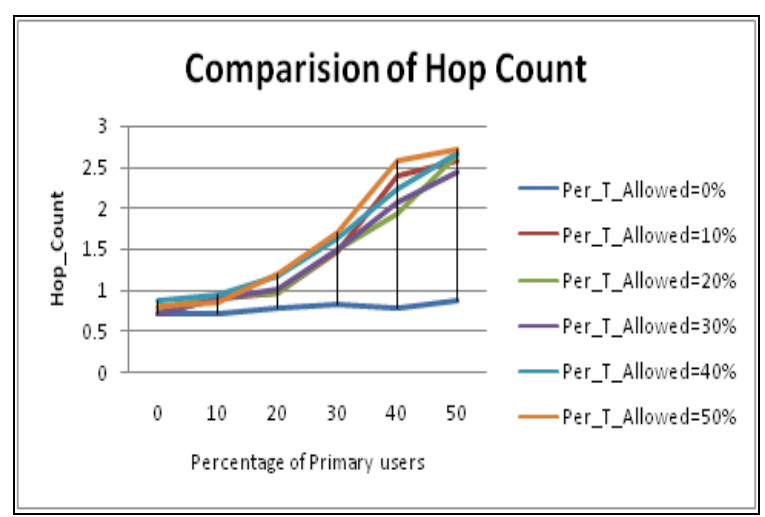

Figure 3.7. Impact on Hop Count for $15 \%$ size obstacle situation

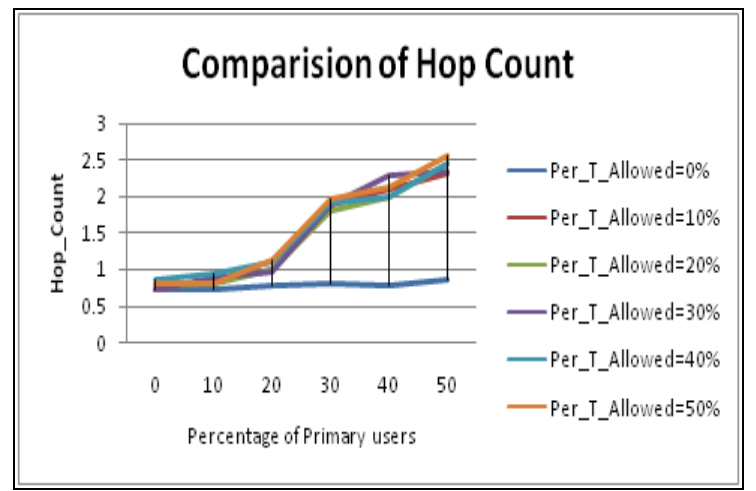

Figure 3.8. Impact on Hop Count for $20 \%$ size obstacle situation

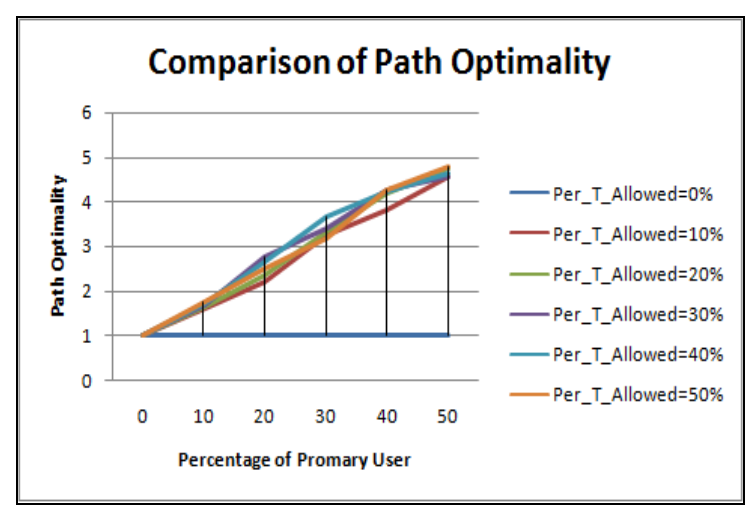

Figure 3.9. Impact on Path Optimality for 5\% size obstacle situation. 


\section{Analysis of Experiment 3}

The overall comparison of the performance metrics with the variation in size of obstacle is shown in Table 4.

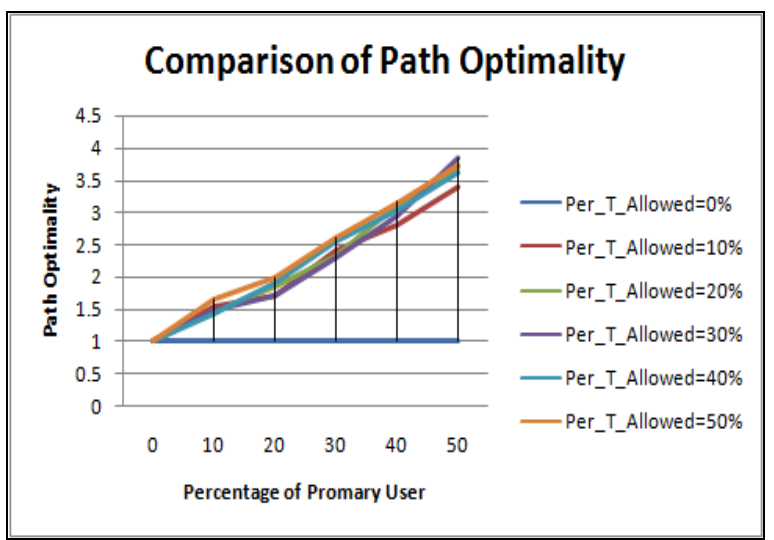

Figure 3.10. Impact on Path Optimality for $10 \%$ size obstacle situation.

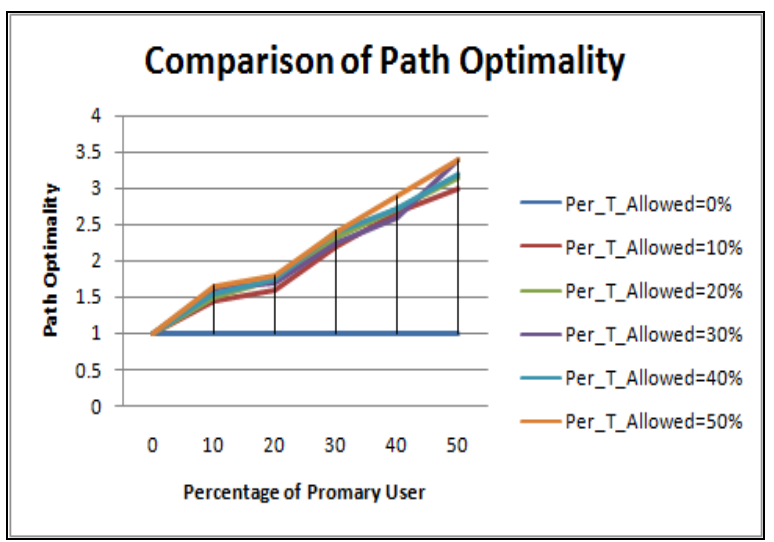

Figure 3.11. Impact on Path Optimality for $15 \%$ size obstacle situation.

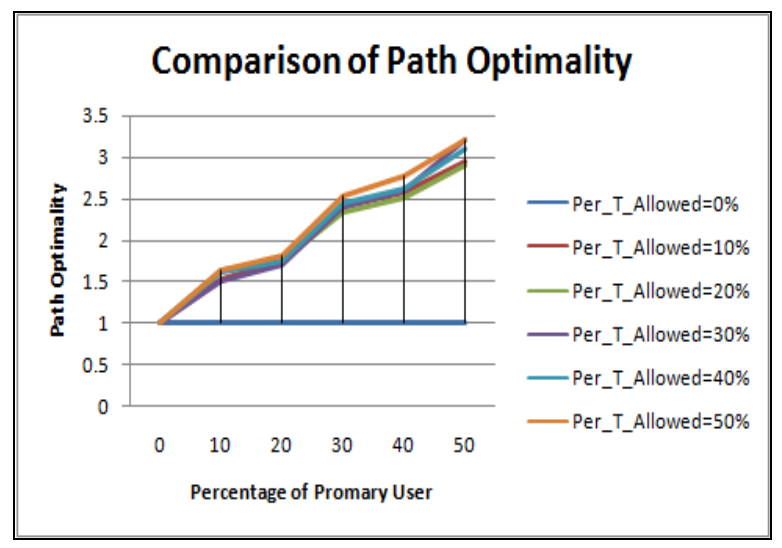

Figure 3.12. Impact on Path Optimality for $20 \%$ size obstacle situation
TABLE 4: OVERALL COMPARISON OF EXPERIMENT 3

\begin{tabular}{|c|c|c|c|c|}
\hline $\begin{array}{c}\text { Performance } \\
\text { Metrics } \\
\text { Obstacle }\end{array}$ & $5 \%$ & $10 \%$ & $15 \%$ & $20 \%$ \\
\hline PDR & & & & \\
Min & 0.09 & 0.089 & 0.08 & 0.08 \\
Max & 0.18 & 0.17 & 0.16 & 0.15 \\
\hline Path Optimality & 1 & 1 & 1 & 1 \\
Min & 4.8 & 3.9 & 3.5 & 3.1 \\
Max & 1.01 & 0.86 & 0.71 & 0.73 \\
Hop count & 3.49 & 3.05 & 2.72 & 2.56 \\
\hline Min & & &
\end{tabular}

\section{J. Experiment 4}

In this experiment an effort has been made to find the Impact on the performance metrics by changing the shapes of the simulation region. In addition to it we also incorporated obstacles to evaluate the network performance. Figure 4(a) to Figure 4(h) shows the snapshots of the experiment performed.

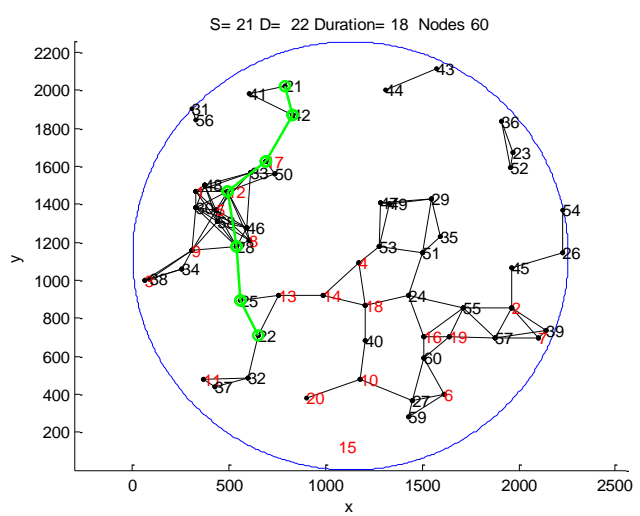

Figure 4(a). Circular shape without obstacle

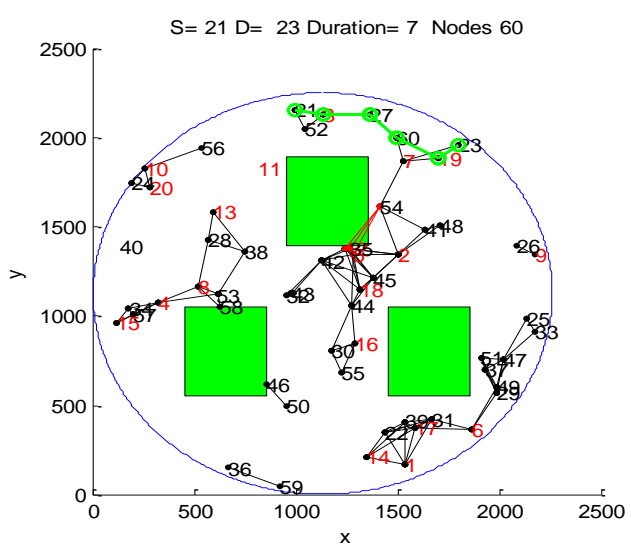

Figure 4(b). Circular shape with obstacle 


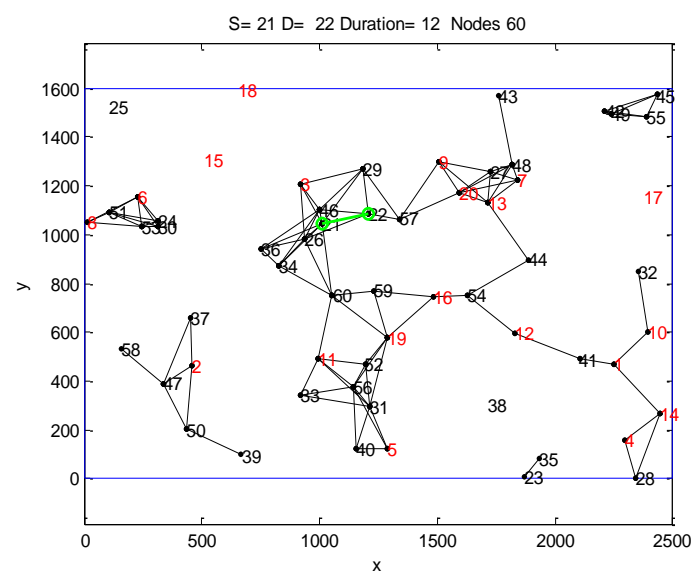

Figure 4(c). Rectangle shape without obstacle

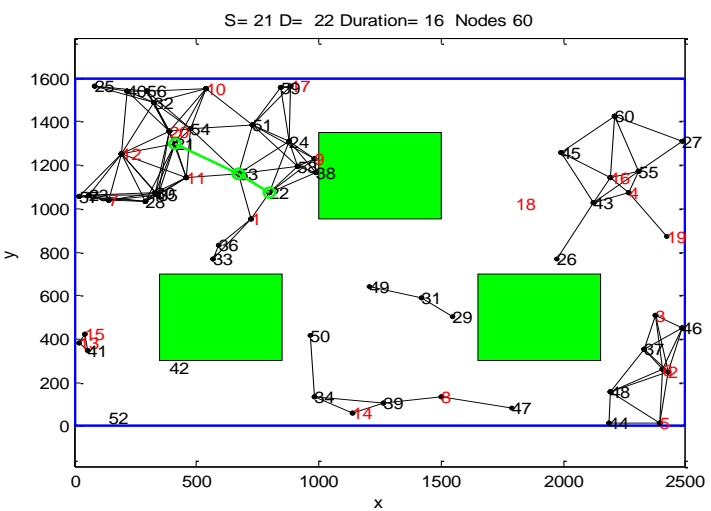

Figure 4(d). Rectangle shape with obstacle

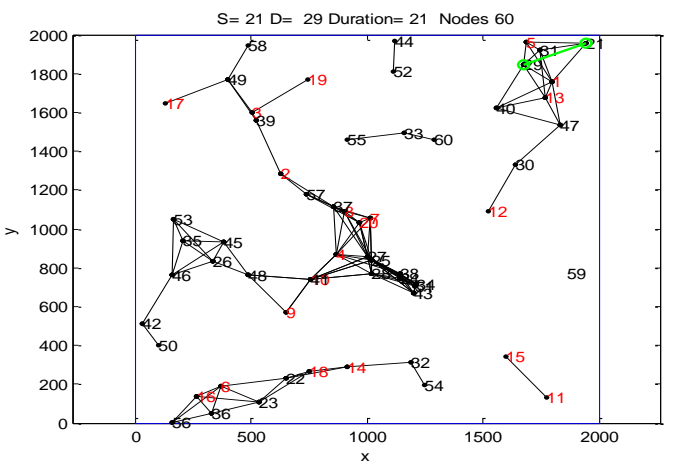

Figure 4 (e). Square region without obstacles

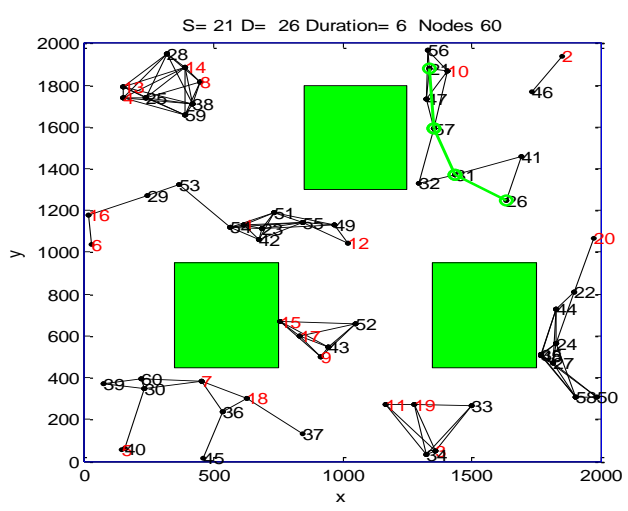

Figure 4 (f) Square region with obstacle

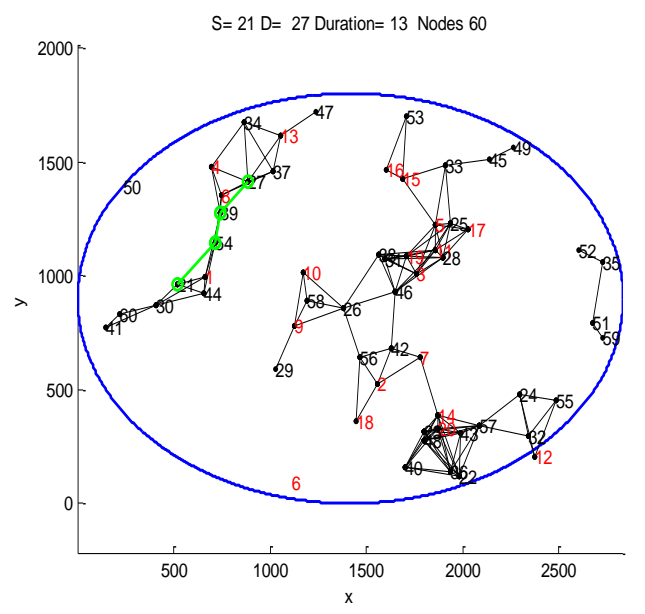

Figure 4(g). Elliptical region without obstacle

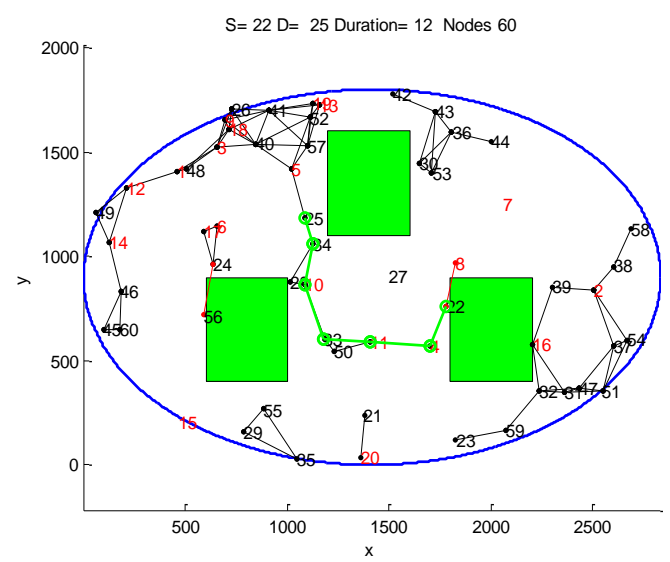

Figure 4(h). Elliptical region with obstacle

\section{J.1 PDR Comparison}

Following inferences about PDR can be drawn from Figure 4.1 to 4.8

- In case of idealistic scenario i.e. in absence of obstacle the value of PDR is comparatively higher for all the shapes of the periphery. This shows that idealistic results are quite different from realistic ones. Thus before choosing any routing protocol the geographical shape of the region should be considered to have better picture of realistic situation.

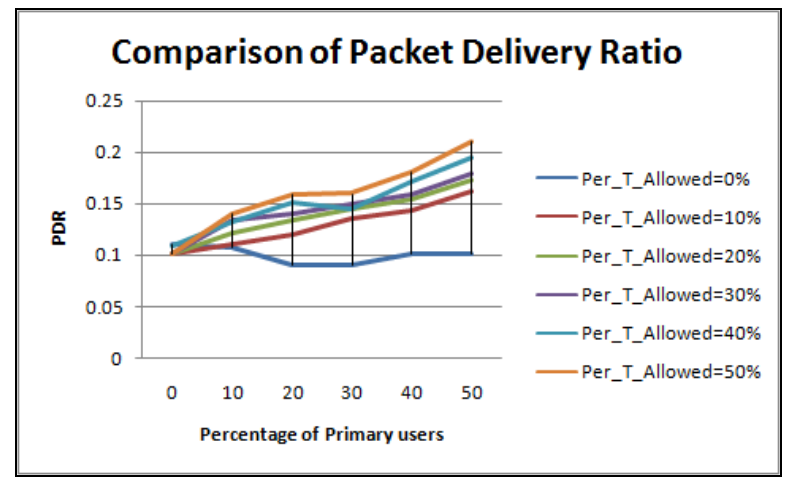

Figure 4.1. Impact on PDR in circular boundary without obstacle situation 


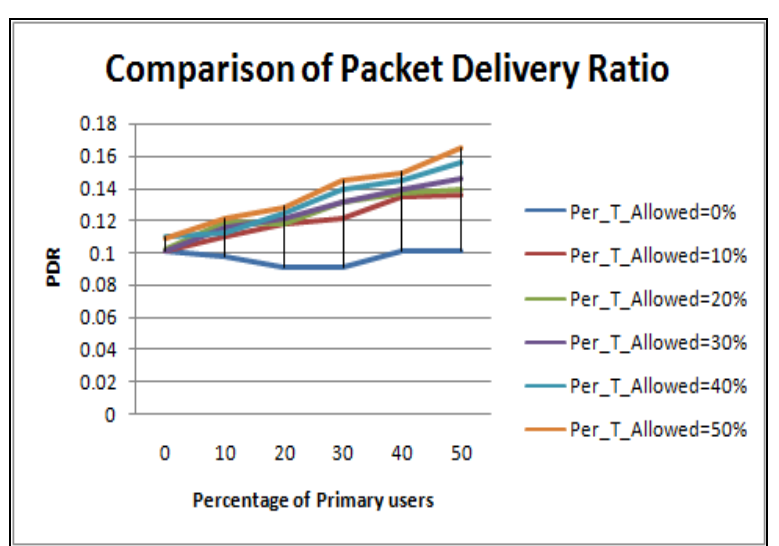

Figure 4.2. Impact on PDR in circular boundary with obstacle situation

- Circular shape region has highest value of PDR value due to symmetry in deployment of nodes in circular region followed by square, rectangle and then elliptical peripheries.

- Change in the PDR when PU concentration is changed from $0 \%$ to 10 to $20 \%$ is quite small in scenario having obstacle.

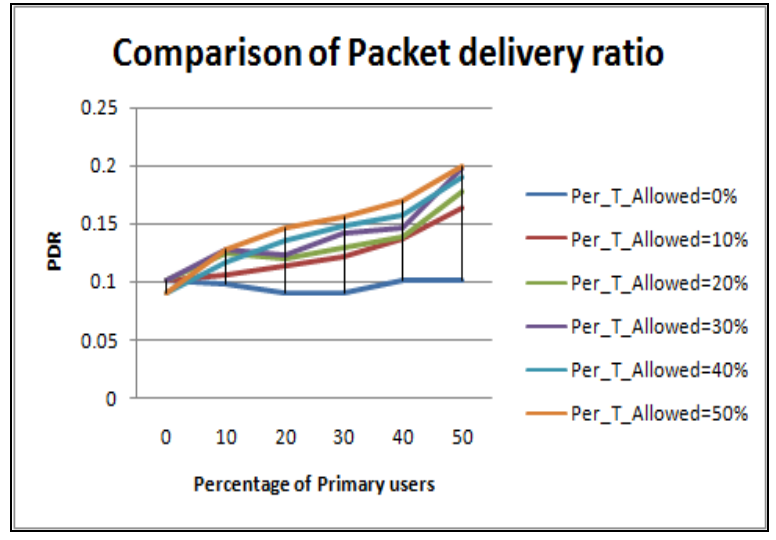

Figure 4.3. Impact on PDR in Rectangular boundary without obstacle situation

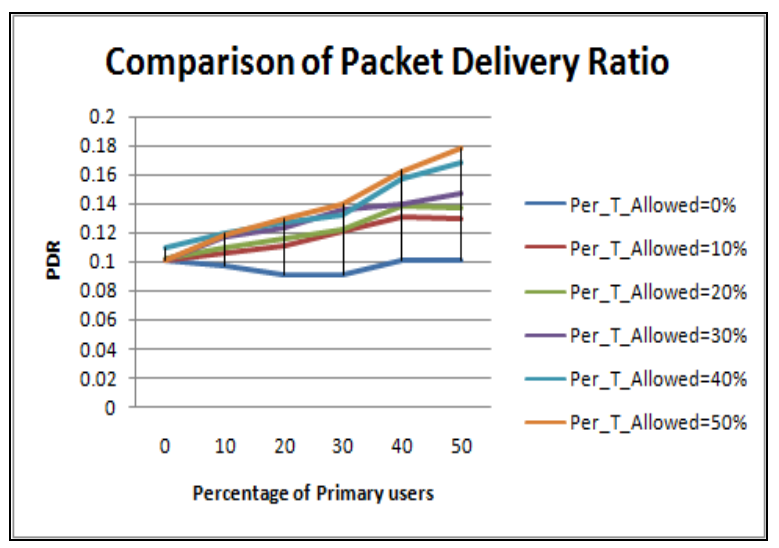

Figure 4.4. Impact on PDR in Rectangular boundary with obstacle

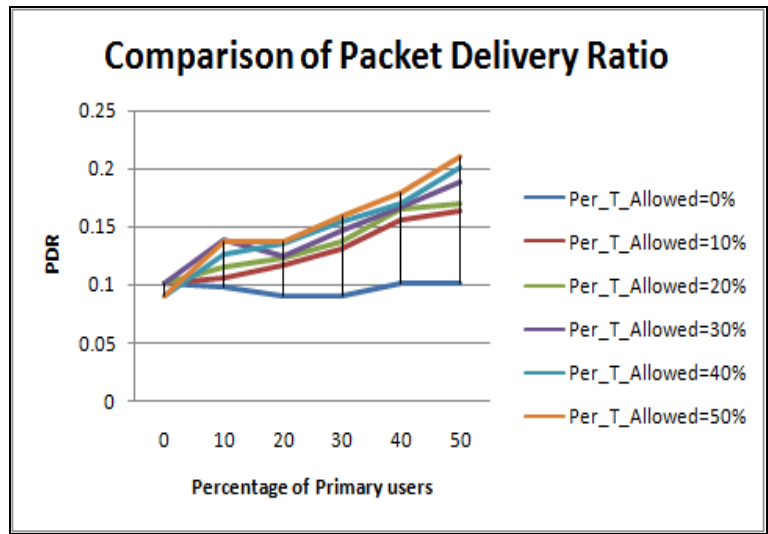

Figure 4.5. Impact on PDR in square boundary without obstacle situation

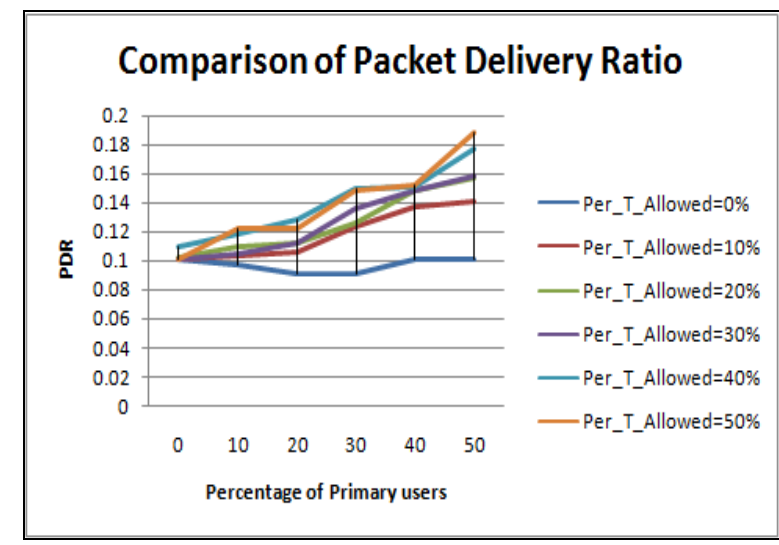

Figure 4.6. Impact on PDR in square boundary with obstacle situation

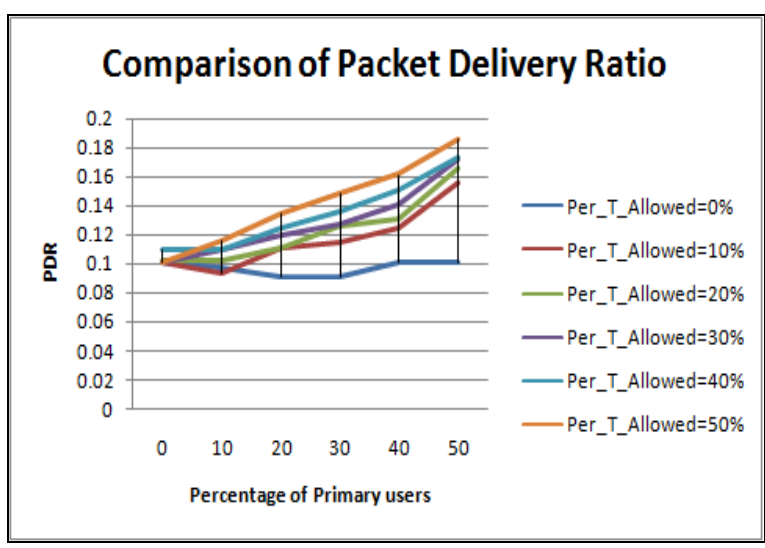

Figure 4.7. Impact on PDR in Elliptical boundary without obstacle situation 


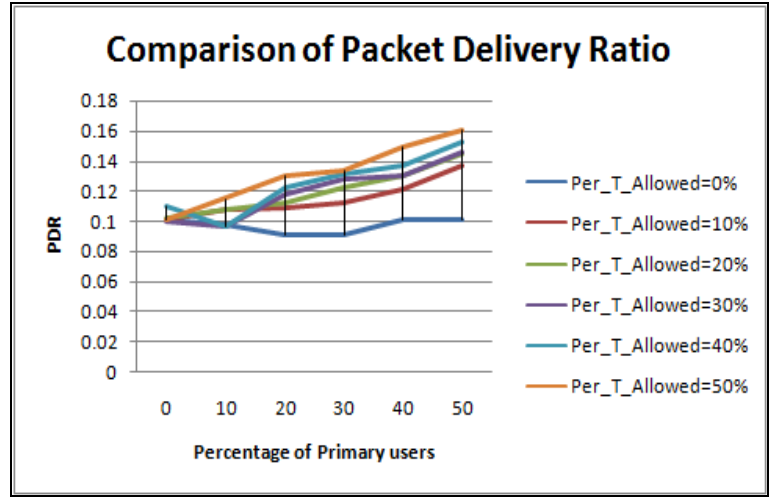

Figure 4.8. Impact on PDR in Elliptical boundary with obstacle situation

\section{J.2 Hop Count Comparison}

Figure 4.9 to Figure 4.16 shows the hop count for different shape of the periphery, following inference can be drawn:

- As discussed the circle has maximum value of PDR therefore its hop count must be least as also depicted from our results.

- As discussed the elliptical shape of the periphery and rectangular shape has same value of line of symmetry hence the results of hop count are nearly same.

- The realistic results are quite different from the idealistic ones as also proven above.

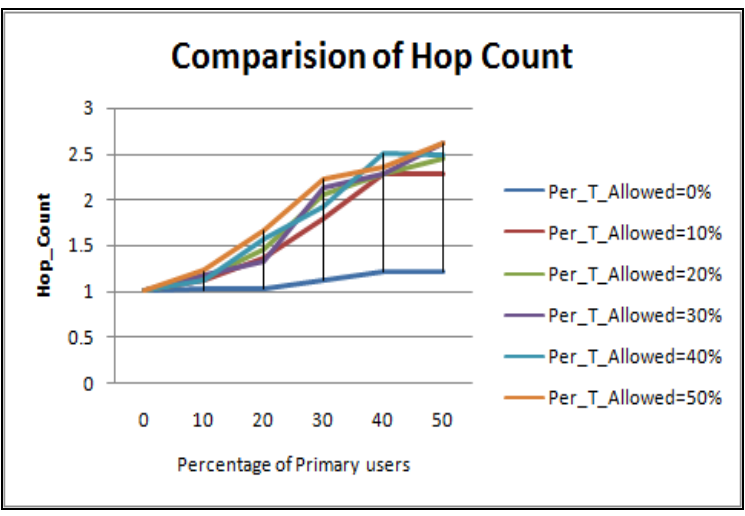

Figure 4.9. Impact on Hop Count in circular boundary without obstacle situation

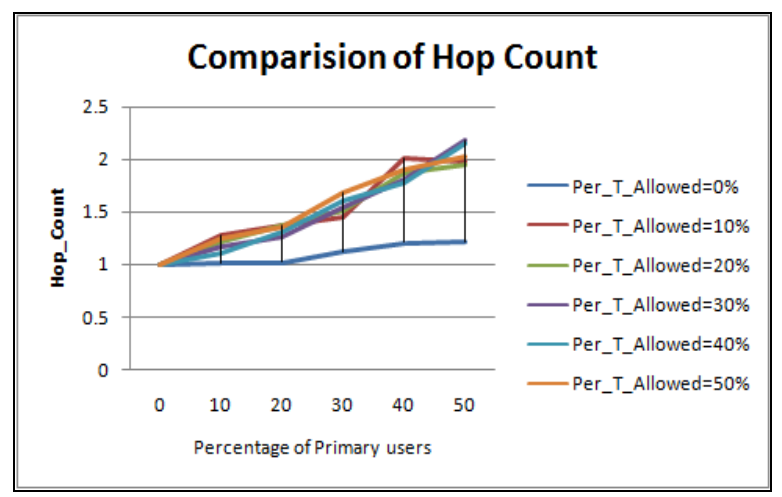

Figure 4.10. Impact on Hop Count in circular boundary with obstacle situation

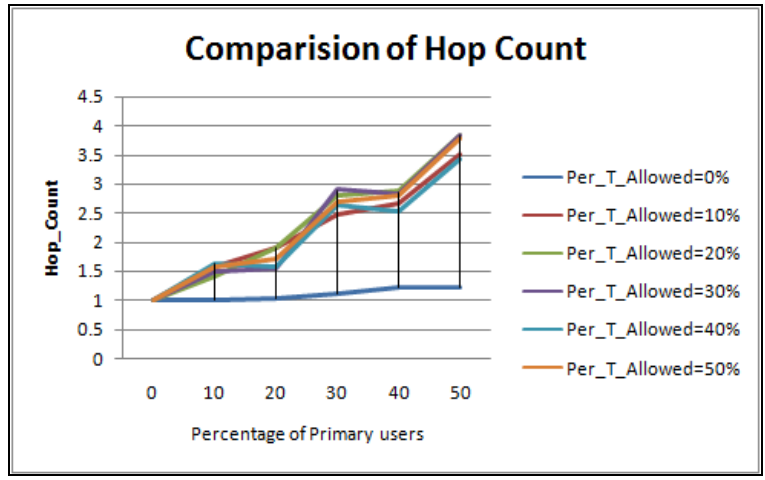

Figure 4.11. Impact on Hop Count in Rectangular boundary without obstacle situation

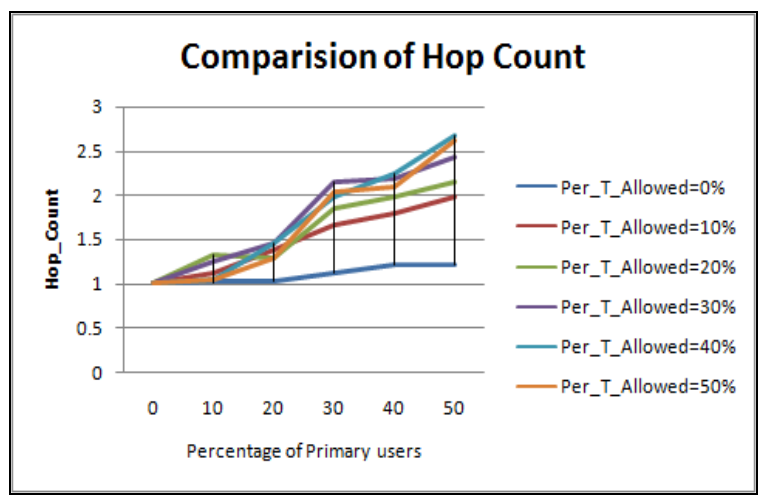

Figure 4.12. Impact on Hop Count in Rectangular boundary with obstacle situation

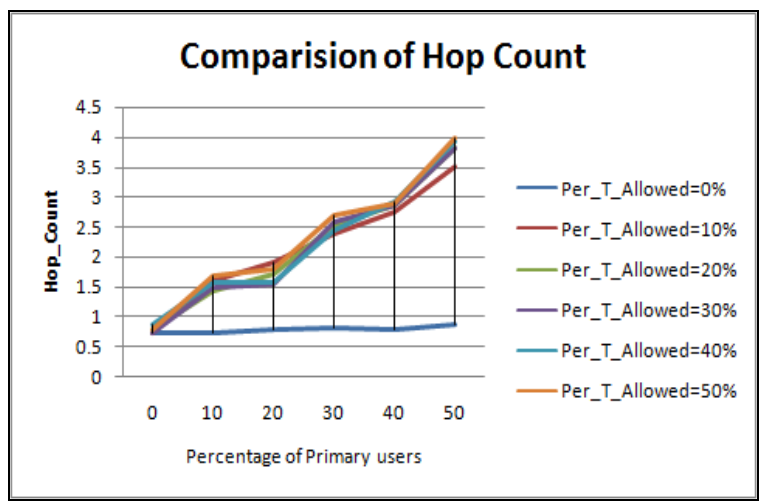

Figure 4.13. Impact on Hop Count in square boundary without obstacle situation

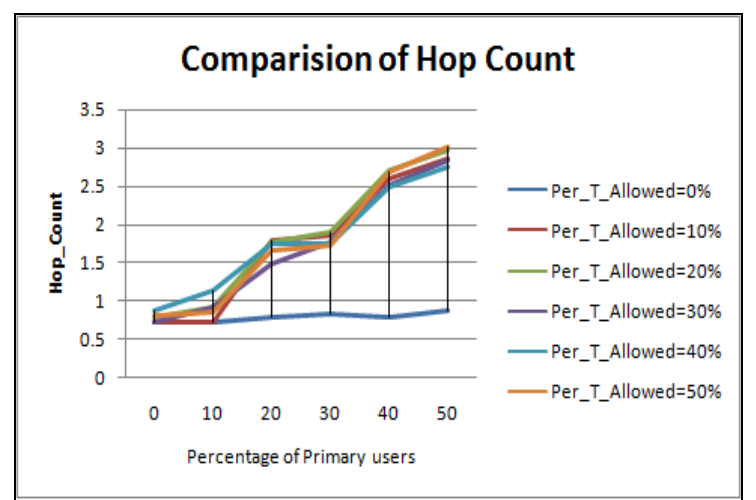

Figure 4.14. Impact on Hop Count in square boundary with obstacle situation 


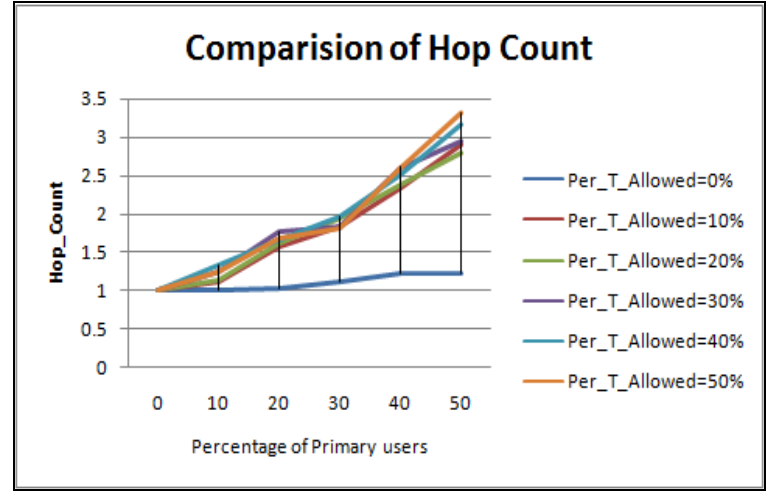

Figure 4.15. Impact on Hop Count in Elliptical boundary without obstacle situation

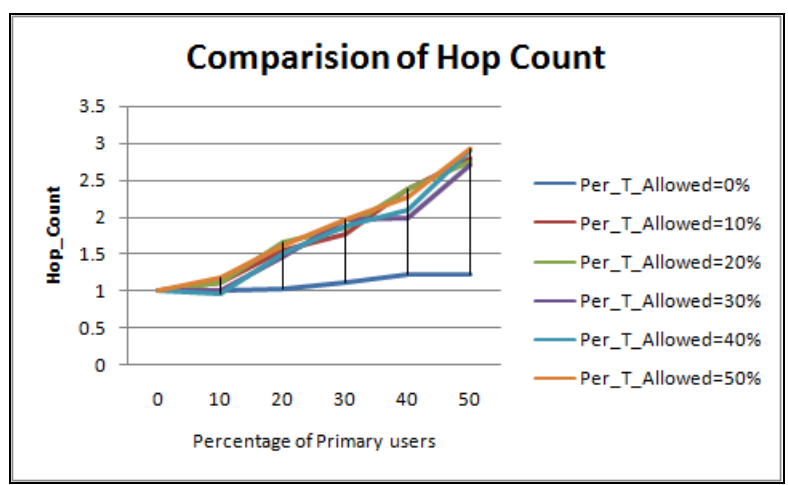

Figure 4.16. Impact on Hop Count in Elliptical boundary with obstacle situation

\section{J.3. Path Optimality Comparison}

Figure 4.17 to Figure 4.24 shows the path optimality for different shape of the periphery, Following inference can be drawn:

- As discussed the circle has maximum value of PDR therefore its path length and hop count must be least as also depicted from our results.

- As discussed the elliptical shape of the periphery and rectangular shape has same value of line of symmetry hence the results of hop count are nearly same..

- The realistic results are quite different from the idealistic ones as also proven above.

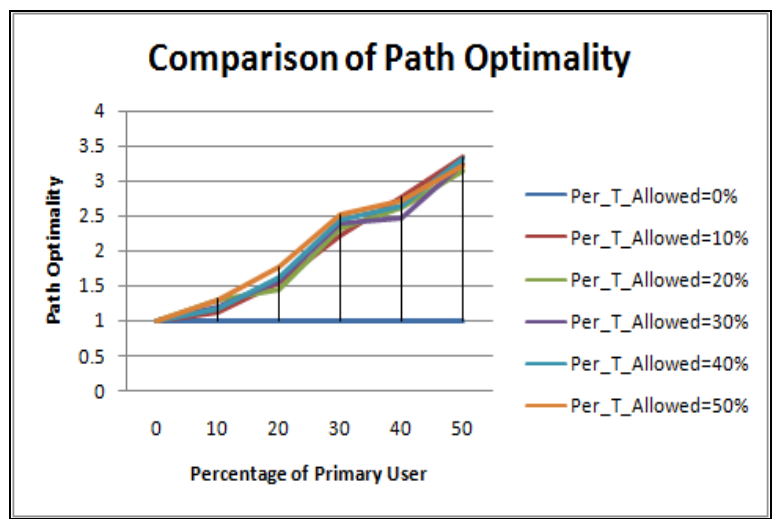

Figure 4.17. Impact on Path Optimality in circular boundary without obstacle situation

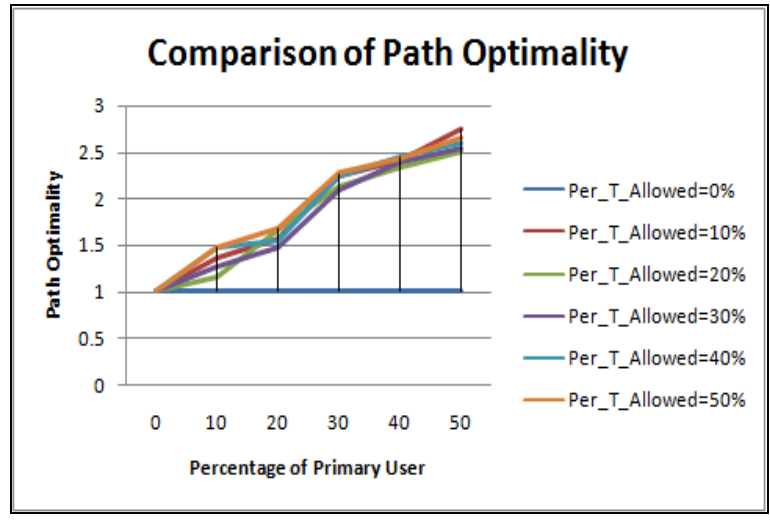

Figure 4.18. Impact on Path Optimality in circular boundary with obstacle situation

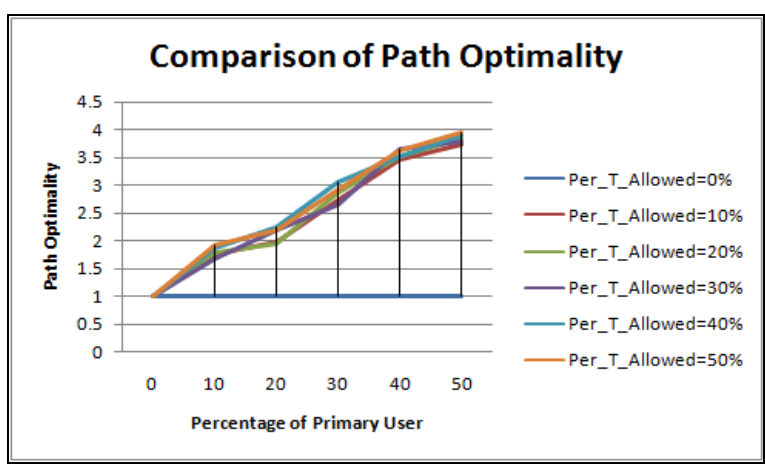

Figure 4.19. Impact on Path Optimality in Rectangular boundary without obstacle situation

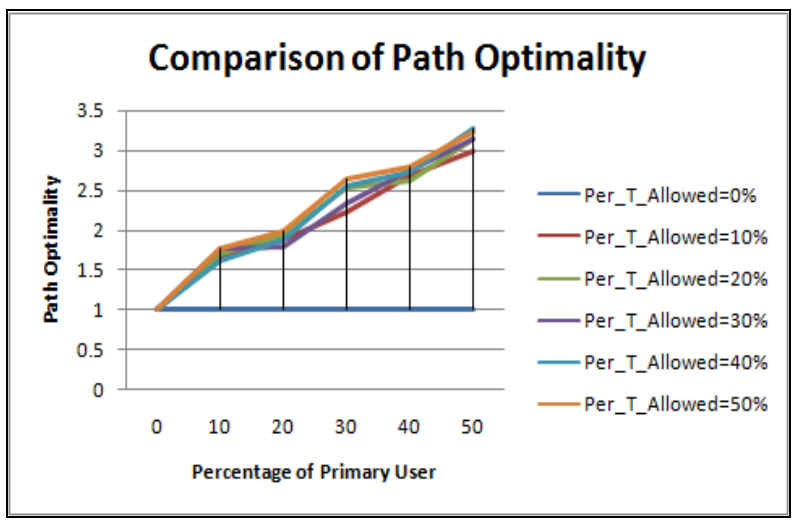

Figure 4.20. Impact on Path Optimality in Rectangular boundary with obstacle situation

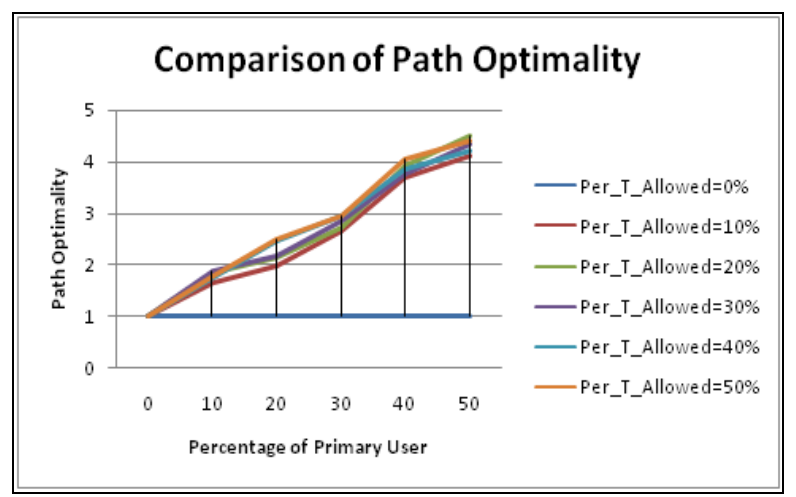

Figure 4.21. Impact on Path Optimality in square boundary without obstacle situation 


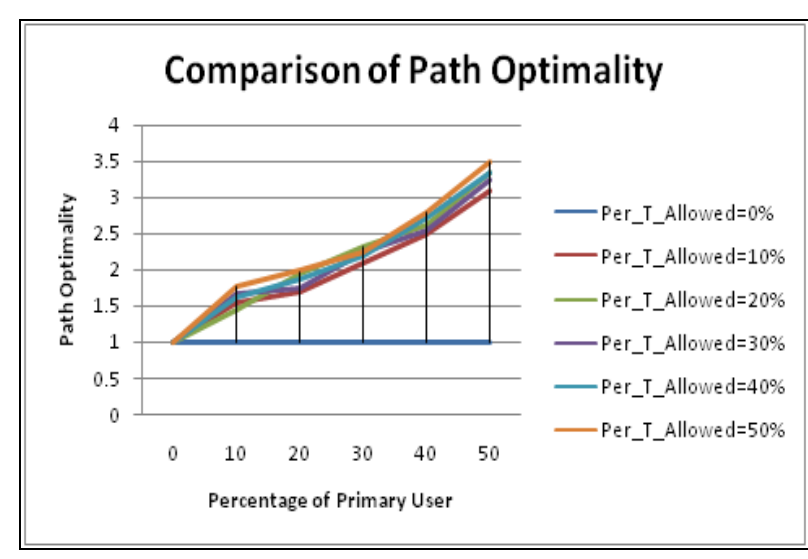

Figure 4.22. Impact on Path Optimality in square boundary with obstacle situation

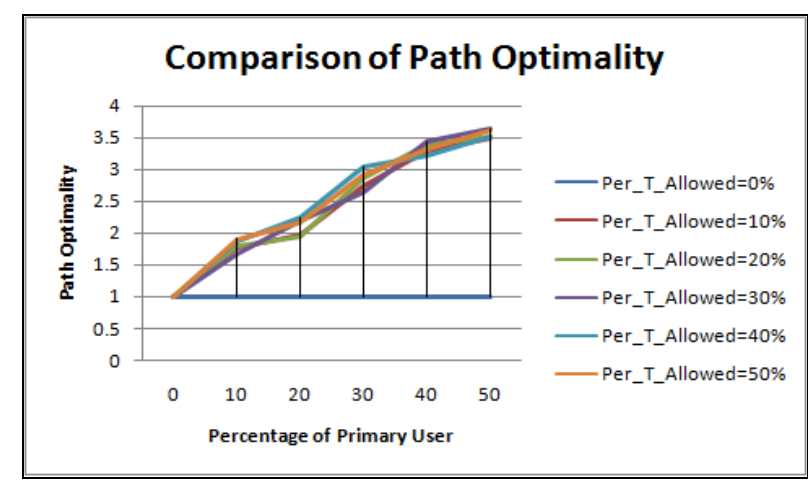

Figure 4.23. Impact on Path Optimality in Elliptical boundary without obstacle situation

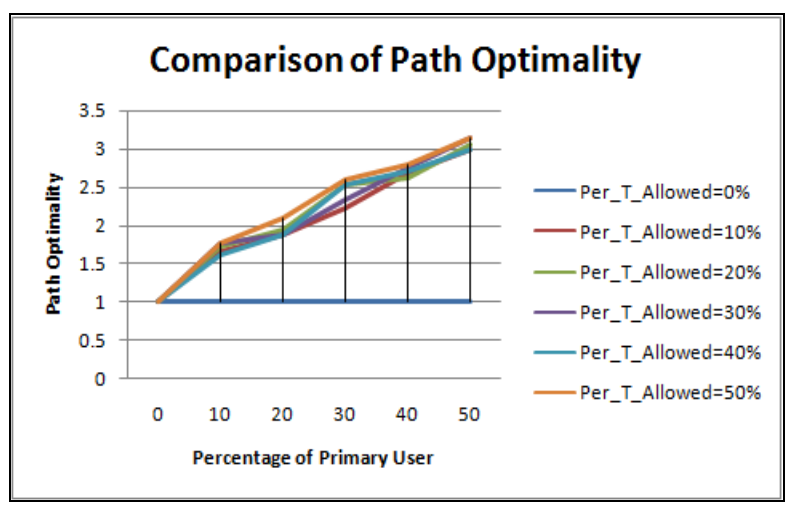

Figure 4.24. Impact on Path Optimality in Elliptical boundary with obstacle situation

\section{K. Analysis of Experiment 4}

From this experiment it can be easily seen that if the geographical shape is circular all the performance metrics proves to be the best. The reason behind this is the fact the circular region has infinite lines of symmetry. For the other shape i.e. square which has four lines of symmetry has the best performance when compared with rectangle and ellipse. The other two shapes have same lines of symmetry that's why their results are nearly same as also shown in Table5.

\section{CONCLUSION}

In this paper an effort has been made to make the scenario realistic for CRN. Following are the outcomes of this paper that can prove very much beneficial for researchers:

(1) Realistic results obtained are quite different from ideal ones as shown. Thus they cannot be used in actual scenarios of deployment of CRN.

(2) Before choosing any protocol for simulations the geographical shape of the network must be taken into consideration since different shape of the simulation region have different impact on performance of routing protocols.

(3) The behaviour of routing protocol is quite different in the simulation region having different type of obstacles.

The paper also shows how crn can solve our network scarcity problem.

Table 5: Overall Comparison of Experiment 4

\begin{tabular}{|c|c|c|c|c|c|c|}
\hline \multirow{3}{*}{$\begin{array}{c}\text { Performance } \\
\text { Metrics } \\
\text { Shape of } \\
\text { Boundary } \rightarrow\end{array}$} & \multirow{3}{*}{$\begin{array}{c}\text { Circle } \\
\text { Without With } \\
\text { Obstacle Obstacle }\end{array}$} & Square & \multicolumn{2}{|c|}{ Ellipse } & \multicolumn{2}{|c|}{ Rectangle } \\
\hline & & Without With & Without & With & Withou & t With \\
\hline & & Obst . & Obst . & Obst. & Obst. & Obst. \\
\hline Lines of Symmetry & Infinite & 4 & & & & 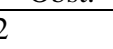 \\
\hline Min & $0.11067 \quad 0.10131$ & $\begin{array}{lll}0.10131 & 0.10067\end{array}$ & 0.10067 & 0.10965 & 0.09912 & 0.91233 \\
\hline PDR & $0.21164 \quad 0.16545$ & $0.21007 \quad 0.18797$ & 0.18523 & 0.16062 & 0.20076 & 0.17864 \\
\hline Path & 1 & 1 & 1 & 1 & 1 & 1 \\
\hline Optimality Max & 3.21 & 3.48 & 3.62 & 3.14 & 3.98 & 3.24 \\
\hline Min & 1.012 & $0.86705 \quad 0.8056$ & 1.023 & 0.923 & 0.923 & 1.011 \\
\hline Hop count Max & 2.632 & $3.853 \quad 3.0091$ & 3.326 & 2.930 & 3.767 & 2.618 \\
\hline
\end{tabular}




\section{REFERENCES}

[1] Shailender Gupta, C.K Nagpal, Charu Singla, "Impact of selfish node concentration in Manets" Published in International Journal of Wireless and Mobile Networks (IJWMN), Vol.3, No.2,pp.29-37, April 2011

[2] Ying -Chang Liang, kwang Cheng Chen and Geoffrey, "Cognitive Radio Networking and communications: An overview", Published in IEEE Transactions on Vehicular Technology, Vol.60, No.7, Sep2011.

[3] S.M Kamruzzaman, Eunhee Kim, DongGeun Jeong, "An Energy Efficient QoS Routing Protocol for cognitive radio and Ad Hoc Networks", Published in International Conference on Advance Communication technology(ICACT) pp.344349,2011.

[4] Y.-S Chen and S.H. Liao, "Spectrum-aware Routing in discontinuous orthogonal Frequency division multiplexing-based CR Ad Hoc networks". Published in IET Networks,2012, Vol.1pp.20-33.

[5] A.C. Talay and D.T. Altilar, "United Nodes: Cluster based routing protocol for mobile Cognitive radio networks", Published in IET Communications. Vol. 5, Iss. 15, pp. 2097-2105 Turkey, June 2011.

[6] D.Datla, A.M Wyglinski, and G.J. Minden, “ A spectrum surveying framework for dynamic spectrum access networks, "Published in IEEE trans, Veh. Technal., vol 58, no.8 pp.4158-4168,oct.2009

[7] J. Mitola and G.Q.Maguire ,“ Cognitive radio: making software radios more personal," Published in IEEE Pers. Comm., vol.6, no.4,pp.13-18,aug.1999 .

[8] Amit jardosh, elizabeth m. beldingroyer,kevin c. almeroth,subhash suri,"towards realistic mobility models for mobile adhoc networks", Published MobiCom'03, September 14-19, 2003, San Diego, California, USA.

[9] Natrajan Meghnathan, "Impact of Range of Simulation Time and Network Shape on Hop Count and Stability of Routes in Mobile Adhoc Networks", Published in IAENG: International Journal of Computer Science, 36: 1, IJCS_36_1_01.

[10] J. Mitola , “ Cognitive radio -An integrated agent architecture for software defined radio," Ph.D. dissertation, Roy. Inst. Technol., Stockholm, Sweden, May, 2000.

[11]S. haykin, " Cognitive radio: Brain empowered wireless communication," Published in IEEE J .Sel. Areas Commun., Vol.23, no.2,pp.201-220, feb.2005

[12]F. Akyildiz, W. Y. Lee, M. Vuran and S. Mohanty, "Next generation/dynamic spectrum access/ cognitive radio wireless networks: A survey," published in Computer Networks: The International Journal of Computer and Telecommunications Networking., vol. 50, no. 13 ,pp.2127-2159, sep.2006.

[13] A Sampath, L. Yang, L. Cao, H. Zheng and B. Y Zhao, " high Throughput spectrum -aware routing for cognitive radio networks," Published in Third
International Conference on Cognitive Radio Oriented Wireless Networks and Communications (CROWNCOM), 2008.

[14] T. Fuji and Y. Yamao, "Multi-band routing for adhoc cognitive radio networks," Published in Proc. SDR Tech. Conf., Nov.2006.

[15]H. Ma, L. Zheng, X. Ma and Y. Luo, "Spectrum aware routing for multi-hop cognitive radio Networks with a single transceiver" Published in Cognitive radio oriented Wireless Networks and communication.(Crown Com),3rd International Conference, may 2008.

[16] K.-C. Chen, B. K. Cetin, Y.-C. Peng, N.Prasad, J.Wang, and S.Lee, "Routing for Cognitive radio Networks consisting of opportunistic links," Published in Wirel. Commumn.Mob.Comput., Vol.10, no .4,pp.451-4566,Apr. 2010.

[17]Zhenhui Zhai, Yong Zhang, Mei Song, Guangquan Chen, " A Reliable \& Adaptive AODV protocol based on Cognitive Routing for ad hoc networks" Published in International Conference on Advance Communication technology(ICACT), 2010.

[18] Sher, Mohammed; Afzal, Muhammad khalil, "Efficient delay and energy based routing in Cognitive radio ad hoc networks". Published in International Conference on Emerging Technologies (ICET) 2012.

[19]El Masri, Ali, “A Fuzzy-based Routing Strategy for Multihop Cognitive Radio Networks" Published in COCORA 2011: The First International Conference on Advances in Cognitive Radio1.

[20] Ashwin Sampath, Lei Yang, Lili Cao, Haitao Zheng, Ben Y. Zhao, "High Throughput Spectrum-aware Routing for Cognitive Radio Networks". Published in proc. of international conference on cognitive radio oriented wireless networks and communications (crowncom), 2007.

Vivek Kukreja received his M.Tech in Electronics and Communication Engineering from YMCA University of Science and Technology, Faridabad, India in 1012.. His interest include ad hoc network.

Shailender Gupta, Received his B.Tech, M.Tech and Ph.D degrees from YMCA University of Science and Technolgy, Faridabad, India. He is faculty in Electronics Engineering Department in YMCA University.. His interests include networking, security and digital signal processing.

Bharat Bhushan received his B.Tech from Punjab Engineering College, Chandigarh, India and M.Tech from YMCA University of Science and Technolgy, Faridabad, India. He is faculty in Electronics Engineering Department in YMCA University. His interest include MANET and DSP. 